ARTICLE OPEN

\title{
Two and three-uniform states from irredundant orthogonal
}

\section{arrays}

\author{
Shan-Qi Pang $\mathbb{D}^{1}$, Xiao Zhang ${ }^{1}$, Xiao Lin $^{1}$ and Qing-Juan Zhang ${ }^{1}$
}

\begin{abstract}
A pure quantum state of $N$ subsystems, each with $d$ levels, is said to be $k$-uniform if all of its reductions to $k$ qudits are maximally mixed. Only the uniform states obtained from orthogonal arrays (OAs) are considered throughout this work. The Hamming distances of OAs are specially applied to the theory of quantum information. By using difference schemes and orthogonal partitions, we construct a series of infinite classes of irredundant orthogonal arrays (IrOAs), then answer the open questions of whether there exist 3-uniform states of $N$ qubits and 2-uniform states of $N$ qutrits, and whether 3-uniform states of qudits $(d>2)$ for high values of $N$ can be explicitly constructed. In fact, we obtain 3-uniform states for an arbitrary number of $N \geq 8$ qubits and 2uniform states of $N$ qutrits for every $N \geq 4$. Additionally, we provide explicit constructions of the 3-uniform states of $N \geq 8$ qutrits, $N=6$ and $N \geq 8$ ququarts and ququints, $N \geq 6$ qudits having $d$ levels for any prime power $d>6$, and $N=8$ and $N \geq 12$ qudits having $d$ levels for non-prime-power $d \geq 6$. Moreover, we describe an explicit construction scheme for the 2-uniform states of qudits having $d \geq 4$ levels. The proofs of existence of the 2-uniform states of $N \geq 6$ qubits are simplified by using a class of OAs. Two special 3uniform states are obtained from $\operatorname{IrOA}(32,10,2,3)$ and $\operatorname{IrOA}(32,11,2,3)$ using the interaction column property of OAs.
\end{abstract}

npj Quantum Information (2019)5:52; https://doi.org/10.1038/s41534-019-0165-8

\section{INTRODUCTION}

Multi-particle entanglement is an essential component in describing the possible quantum advantages available to metrology or information processing. The identification of multipartite quantum states with the strongest possible quantum correlations is a crucial question in quantum information theory. ${ }^{1}$ An important open issue concerns the construction of genuinely multipartite entangled states, ${ }^{2}$ as these have been widely applied to quantum errorcorrecting codes (QECCs), ${ }^{3,4}$ teleportation, ${ }^{5-8}$ key distribution, ${ }^{9}$ dense coding, and quantum computation. ${ }^{10}$ For example, in the past few years, significant development has been made in the new area of quantum machine learning, where quantum information benefits from modern information-processing technologies. ${ }^{11}$ Quantum entanglement as a resource has been used to experimentally demonstrate various modern quantum technologies.

A pure quantum state of $N$ subsystems with $d$ levels is said to be $k$-uniform if all of its reductions to $k$ qudits are maximally mixed. ${ }^{2}$ An orthogonal array $\mathrm{OA}\left(r, N, d_{1}^{n_{1}} d_{2}^{n_{2}} \cdots d_{1}^{n_{1}}, k\right)$ is an $r \times N$ matrix, having $n_{i}$ columns with $d_{i}$ levels, $i=1,2, \ldots, l, l$ is an integer, $N=\sum_{i=1}^{l} n_{i}$, and $d_{i} \neq d_{j}$ for $i \neq j$, with the property that, in any $r \times k$ submatrix, all possible combinations of $k$ symbols appear equally often as a row. The orthogonal array is called a mixed orthogonal array (MOA) if $I \geq 2$. Otherwise, the array is called symmetrical. Additionally, the following Rao bounds hold in $\mathrm{OA}(r, N, d, k)$ :

$r \geq \sum_{i=0}^{\frac{k}{2}}\left(\begin{array}{c}N \\ i\end{array}\right)(d-1)^{i}$, if $k$ is even,

$r \geq \sum_{i=0}^{\frac{k-1}{2}}\left(\begin{array}{c}N \\ i\end{array}\right)(d-1)^{i}+\left(\begin{array}{c}N-1 \\ \frac{k-1}{2}\end{array}\right)(d-1)^{\frac{k+1}{2}}$, if $k$ is odd.
OAs that achieve these bounds are called tight or saturated. ${ }^{12}$

An $\mathrm{OA}(r, N, d, k)$ is said to be an irredundant orthogonal array (IrOA) if, in any $r \times(N-k)$ subarray, all of its rows are different. ${ }^{2} A$ link between an IrOA and a $k$-uniform state was established by Goyeneche et al., ${ }^{2}$ i.e., every column and every row of the array correspond to a particular qudit and a linear term of the state, respectively. For simplicity, the normalization factors are omitted from this paper. We summarize the link as follows:

Connection 1.1.

${ }^{2}$ If $L=\left(\begin{array}{cccc}s_{1}^{1} & s_{2}^{1} & \cdots & s_{N}^{1} \\ s_{1}^{2} & s_{2}^{2} & \cdots & s_{N}^{2} \\ \vdots & \vdots & \cdots & \vdots \\ s_{1}^{r} & s_{2}^{r} & \cdots & s_{N}^{r}\end{array}\right)$ is an $\operatorname{IrOA}(r, N, d, k)$, then the superposition of $r$ product states, $|\Phi\rangle=\left|s_{1}^{1} s_{2}^{1} \ldots s_{N}^{1}\right\rangle+\left|s_{1}^{2} s_{2}^{2} \ldots s_{N}^{2}\right\rangle+\cdots+\left|s_{1}^{r} s_{2}^{r} \ldots s_{N}^{r}\right\rangle$ is a -uniform state.

Many efforts have attempted to find and identify $k$-uniform states. ${ }^{2,4}$ Under the generalizations of the Meyer-Wallach measure defined in, ${ }^{4} k$-uniform states have the largest average entanglement between blocks of qudits and the remaining elements. There has also been some progress in the construction and characterization of $k$-uniform states. ${ }^{1,2,11,13-18}$ For example, Goyeneche et al. ${ }^{2}$ constructed a 3-uniform state of six qubits and a 2uniform state of five qubits by the judicial insertion of some minus signs. The nonexistence of the 3-uniform states of seven qubits is proved in. ${ }^{14}$ Using the above connection, Goyeneche et al. ${ }^{2}$ constructed 2-uniform states for an arbitrary number of $N \geq 6$ qubits using known Hadamard matrices. Yu et al. ${ }^{19}$ constructed a 3-uniform state of eleven qubits via OAs. However, as stated in, ${ }^{2}$ there are many open issues in multipartite quantum systems. Huber et al. ${ }^{14}$ stated that it would be of great interest to settle the problem of whether $N$-qubit states exist in which all $k$-body reduced density are maximally mixed for $k<\left\lfloor\frac{N}{2}\right\rfloor$.

${ }^{1}$ College of Mathematics and Information Science, Henan Normal University, Xinxiang 453007, China

Correspondence: Shan-Qi Pang (shanqipang@126.com)

Received: 1 November 2018 Accepted: 22 May 2019

Published online: 17 June 2019 
The aim of the present study was to solve the open issues raised $\mathrm{in}^{2}$ and the interesting question stated in ${ }^{14}$ using IrOAs, the states related to which are useful and necessary in quantum error correction. The different uniformity of multipartite entangled states reflects different features. One motivation for searching for multipartite states of a higher uniformity is that $k$-uniform states of qudits offer advantages over $k^{\prime}$-uniform states for any $0<k^{\prime}<k$. They are good for increasing the order of the density coding information rate from $d^{k^{\prime}}$ to $d^{k}{ }^{20}$ It is interesting that these states form a natural extension of $\mathrm{N}$-qudit Greenberger-Horne-Zeilinger states, which are 1-uniform. Moreover, a $k$-uniform state is also $k^{\prime}$-uniform. As is often the case, ${ }^{21-23}$ combinatorics can be useful to quantum information theory, and OAs are fundamental ingredients in the construction of other useful combinatorial objects. $^{12}$ The Hamming distance, difference schemes, and orthogonal partitions have been applied to many aspects of constructing OAs. ${ }^{24-27}$ Recently, many new methods of constructing OAs of strength $k$, especially mixed OAs, have been presented, and many new classes of OAs have been obtained. ${ }^{28-33}$ It is these new developments in OAs that suggest the possibility of constructing infinitely many new $k$-uniform states from IrOAs.

In this work, the Hamming distance and minimal distance (MD) of OAs are applied to the theory of quantum information. By using difference schemes and orthogonal partitions, we construct several infinite series of IrOAs, then consider the open issue in ${ }^{2}$ of whether there exist 3-uniform states of $N$ qubits and 2-uniform states of $N$ qutrits, and whether 3 -uniform states of qudits having $d>2$ levels for high values of $N$ can be explicitly constructed. In fact, we present several series of 3-uniform states for an arbitrary number of $N \geq 8$ qubits and 2-uniform states for an arbitrary number of $N \geq 4$ qutrits. Additionally, we provide explicit constructions of 3-uniform states of $N \geq 8$ qutrits, $N=6$ and $N \geq$ 8 ququarts and ququints, $N \geq 6$ qudits having $d$ levels for any prime power $d>6$, and $N=8$ and $N \geq 12$ qudits having $d$ levels for non-prime-power $d \geq 6$. Moreover, we explicitly construct 2uniform states of $N \geq 4$ qudits $(d \notin\{2,6,10\}), N \geq 5$ qudits ( $d=$ 6 ), and $N=4$ and $N \geq 6$ qudits ( $d=10$ ). The proofs of existence of 2-uniform states of $N \geq 6$ qubits are simplified by using a class of OAs. Furthermore, as it has been proved that $\operatorname{IrOA}(r, 7,2,3), \operatorname{IrOA}(r$, $7,3,3), \operatorname{IrOA}(r, 6,3,3), \operatorname{IrOA}(r, 4,6,2), \operatorname{IrOA}(r, 6,2,3), \operatorname{IrOA}(r, 4,2,2)$, and $\operatorname{IrOA}(r, 5,2,2)$ do not exist, the corresponding uniform states can not be obtained. However, two special 3-uniform states are obtained from $\operatorname{IrOA}(32,10,2,3)$ and $\operatorname{IrOA}(32,11,2,3)$ using the interaction column property of OAs.

The following notation, concepts, and lemmas are used in this paper.

Let $A^{T}$ be the transposition of matrix $A$ and $(\mathbf{d})=(0,1, \ldots, d-$ 1) ${ }^{T}$. Let $\mathbf{0}_{r}$ and $\mathbf{1}_{r}$ denote the $r \times 1$ vectors of $0 s$ and $1 s$, respectively. If $A=\left(a_{i j}\right)_{m \times n}$ and $B=\left(b_{i j}\right)_{u \times v}$ with elements from a Galois field with binary operations ( + and $\cdot)$, the Kronecker product $A \otimes B$ and the Kronecker sum $A \oplus B$ are respectively defined as $A \otimes B=\left(a_{i j}\right.$. $B)_{m u \times n v}$ and $A \oplus B=\left(a_{i j}+B\right)_{m u \times n v}$, where $a_{i j}+B$ represents the $u \times$ $v$ matrix with entries $a_{i j}+b_{r s}(1 \leq r \leq u, 1 \leq s \leq v)$. A matrix $A$ can often be identified with a set of row vectors if necessary. Let $\lceil x\rceil$ denote the least integer not less than $x$, and let $\mathcal{A}$ be an additive group of $d$ elements.

Definition 1.1. ${ }^{27}$ Suppose that $\mathbf{a}, \mathbf{b}, \mathbf{c}$ are three columns in an $\mathrm{OA}(\mathrm{r}$, $N, 2,2)$. Then, $\mathbf{c}$ is called the interaction column between $\mathbf{a}$ and $\mathbf{b}$ if $\mathbf{c} \equiv \mathbf{a}+\mathbf{b}$ or $\mathbf{c} \equiv \mathbf{1}_{\mathrm{r}}+\boldsymbol{a}+\boldsymbol{b}(\bmod 2)$.

Definition 1.2. ${ }^{12}$ Let $S^{\prime}=\left\{\left(v_{1}, \ldots, v_{l}\right) \mid v_{i} \in S, i=1,2, \ldots\right.$, l $\}$. The Hamming distance $\mathrm{HD}(\mathbf{u}, \mathbf{v})$ between two vectors $\mathbf{u}=\left(u_{1}, \ldots, u_{1}\right), \mathbf{v}$ $=\left(v_{1}, \ldots, v_{l}\right) \in S^{\prime}$ is defined as the number of positions in which they differ. The minimal distance of a matrix $A$, written $\operatorname{MD}(A)$, is defined to be the minimal Hamming distance between its distinct rows. HD $(A)$ is used to represent all the values of the Hamming distances between two distinct rows of $A$. The matrix $A$ is said to have constant Hamming distance if $\operatorname{HD}(A)$ is constant for any two distinct rows.

Definition 1.3. ${ }^{12}$ An $r \times c$ matrix $D$ with elements from $\mathcal{A}$ is called a difference scheme if it has the property that, for all $i$ and $j$ with $1 \leq i$, $j \leq c, i \neq j$, among the vector differences between the ith and $j$ th columns, every element of $\mathcal{A}$ appears equally often. Such a matrix is denoted as $D(r, c, d)$.

For instance, if $B_{r}$ is an $\mathrm{OA}(r, c-1,2,2)$, then $\left[\boldsymbol{0}_{r}, B_{r}\right]$ is $D(r, c, 2)$. $\mathcal{A}^{k}, k \geq 1$, denotes the additive group of order $d^{k}$ consisting of all $k$-tuples of entries from $\mathcal{A}$ with the usual vector addition as the binary operation. Let $\mathcal{A}_{0}^{k}=\left\{\left(x_{1}, x_{2}, \ldots, x_{k}\right): x_{1}=\cdots=x_{k} \in \mathcal{A}\right\}$. Then, $\mathcal{A}_{0}^{k}$ is a subgroup of $\mathcal{A}^{k}$ of order $d$, and its cosets will be denoted by $\mathcal{A}_{i}^{k}, i=1, \ldots, d^{k-1}-1$.

Definition 1.4. ${ }^{12}$ An $r \times$ c matrix $D$ based on $\mathcal{A}$ is called a difference scheme of strength $k$ if, for every $r \times k$ submatrix, each set $\mathcal{A}_{i}^{k}, i=0$, $1, \ldots, d^{k-1}-1$, is represented equally often when the rows of the submatrix are viewed as elements of $\mathcal{A}^{k}$. Such a matrix is denoted by $D_{k}(r, c, d)$.

For $k=2$, this definition is equivalent to Definition 1.3

Definition 1.5. ${ }^{32}$ Let $A$ be an $\mathrm{OA}\left(r, N, d_{1}^{n_{1}} d_{2}^{n_{2}} \cdots d^{n_{1}}, k\right)$. A set $\left\{A_{1}\right.$, $\left.A_{2}, \ldots, A_{u}\right\}$ of orthogonal arrays $\mathrm{OA}\left(\frac{r}{u}, N, d_{1}^{n_{1}} d_{2}^{n_{2}} \cdots d_{1}^{n_{1}}, k_{1}\right)\left(k_{1} \geq 0\right)$ is defined to be an orthogonal partition of strength $k_{1}$ of $A$ if $\bigcup_{i=1}^{u} A_{i}=$ $A$ and $A_{i} \cap A_{j}=\emptyset$ for $i \neq j$. In particular, when $k_{1}=0, A_{i}$ can be considered as an $\mathrm{OA}\left(\frac{r}{u}, N, d_{1}^{n_{1}} d_{2}^{n_{2}} \cdots d_{1}^{n_{1}}, 0\right)$ for each $1 \leq i \leq u$.

Lemma 1.1. ${ }^{12} \mathrm{An} \mathrm{OA}(r, N, 2,2 u)$ exists for $u \geq 1$ if and only if an $\mathrm{OA}$ $(r, N+1,2,2 u+1)$ exists.

Lemma 1.2. ${ }^{27,34}$ A saturated $\mathrm{OA}(r, N, d, 2)$ has a constant Hamming distance $\frac{r}{d}$.

Lemma 1.3. (1) ${ }^{12}$ If $d$ is a prime and the integers $n \geq m \geq 1$, then a difference scheme $D\left(d^{n}, d^{n}, d^{m}\right)$ exists. (2) ${ }^{35}$ If $d$ is a prime power, then a difference scheme $D_{3}\left(d^{2}, d, d\right)$ exists. (3) ${ }^{35}$ If $d \geq 2$, then $a$ difference scheme $D_{3}\left(d^{2}, 4, d\right)$ exists. (4) ${ }^{36}$ If $d \neq 1(\bmod 4)$, then $a$ difference scheme $D_{k}\left(d^{k-1}, k+1, d\right)$ exists. In particular, $D(d, 3, d)$ exists.

Lemma 1.4. ${ }^{12}$ (1) Let $n \geq 2$. Then an $\mathrm{OA}\left(d^{n}, \frac{d^{n}-1}{d-1}, d, 2\right)$ exists for a prime power $d$. (2) If $d$ is a prime power and $k<d$, there is an $\mathrm{OA}\left(d^{k}\right.$, $d+1, d, k)$.

Lemma 1.5. ${ }^{30}$ If $B_{r}$ is an $\mathrm{OA}(r, N, d, 2)$ and $D(r, c, d)$ is a difference scheme, then $\left[B_{r} \oplus \mathbf{0}_{d}, D(r, c, d) \oplus(\mathbf{d})\right]$ is an $\mathrm{OA}(r d, N+c, d, 2)$. Especially, for any integer $n \geq 2$ and a prime power $d$, there exists an $\mathrm{OA}\left(d^{n+1}, \frac{d^{n+1}-1}{d-1}, d, 2\right)=\left[B_{d^{n}} \oplus \mathbf{0}_{d}, D\left(d^{n}, d^{n}, d\right) \oplus(\mathbf{d})\right] \quad$ where $B_{d^{n}}=\mathrm{OA}\left(d^{n}, \frac{d^{n}-1}{d-1}, d, 2\right)$.

For simplicity, we introduce further notation:
$\left(A_{[1,2, \ldots, u]}, r\right)=\left(\begin{array}{c}A_{1} \otimes 1_{r} \\ A_{2} \otimes 1_{r} \\ \vdots \\ A_{u} \otimes 1_{r}\end{array}\right)$ and $\left(r, A_{[1,2, \ldots, u]}\right)=\left(\begin{array}{c}1_{r} \otimes A_{1} \\ 1_{r} \otimes A_{2} \\ \vdots \\ 1_{r} \otimes A_{u}\end{array}\right)$ for positive integers $u$ and $r$.

Lemma 1.6. ${ }^{32}$ Let $\left\{A_{1}, A_{2}, \ldots, A_{u}\right\}$ be an orthogonal partition of strength $k_{1}$ of $\mathrm{OA}\left(r^{\prime}, N^{\prime}, d, k^{\prime}\right)$, and $\left\{B_{1}, B_{2}, \ldots, B_{v}\right\}$ be an orthogonal partition of strength $k_{2}$ of $\mathrm{OA}\left(r^{\prime \prime}, N^{\prime \prime}, d, k^{\prime \prime}\right)$. Let $h=$ I.c.m. $\{u, v\}$. Then, the array $M=\left(\mathbf{1}_{\frac{h}{u}} \otimes\left(A_{[1,2, \ldots, u]}, \frac{r^{\prime \prime}}{v}\right), \mathbf{1}_{\frac{h}{v}} \otimes\left(\frac{r^{\prime}}{u}, B_{[1,2, \ldots, v]}\right)\right)$ is an OA $\left(\frac{r^{\prime} r^{\prime \prime} h}{u v}, N^{\prime}+N^{\prime \prime}, d, k\right)$, where $k=k_{1}+k_{2}+1 \leq \min \left\{k^{\prime}, k^{\prime \prime}\right\}$. 
Lemma 1.7. ${ }^{32}$ Let $A$ and $B$ be orthogonal arrays with $d$ rows of strength 1. Then $C=\left(A \otimes \mathbf{1}_{d}, \mathbf{1}_{d} \otimes B\right)$ is an orthogonal array of strength 1 and there is an orthogonal partition $\left\{C_{1}, C_{2}, \ldots, C_{d}\right\}$ of $C$ of strength 1.

Lemma 1.8. If $d$ is a prime power, there exists a difference scheme $D$ $(2 d, 2 d, d)$ such that $D(2 d, 2 d, d)^{T}=D(2 d, 2 d, d)$.

Lemma 1.9. ${ }^{37}$ If the transposition of a difference scheme $D(\lambda d, \lambda d$ d) is still a difference scheme, then $\operatorname{HD}(D(\lambda d, \lambda d, d))=\lambda d-\lambda$, and the $\mathrm{OA}\left(\lambda d^{2}, \lambda d, d, 2\right)=D(\lambda d, \lambda d, d) \oplus(\mathbf{d})$ has two Hamming distances $\lambda d$ and $\lambda d-\lambda$. Especially, if $D(2 d, 2 d, d)^{T}=D(2 d, 2 d, d)$, the $\mathrm{OA}\left(2 d^{2}, 2 d\right.$, $d, 2)=D(2 d, 2 d, d) \oplus(\mathbf{d})$ has two Hamming distances $2 d$ and $2 d-2$.

Lemma 1.10. ${ }^{38}$ For any positive integer $d \geq 2$, the following properties hold: (1) If $d \notin\{2,6\}$, then an $\mathrm{OA}\left(d^{2}, 4, d, 2\right)$ exists. (2) If $d$ $\notin\{2,3,6,10\}$, then an $\mathrm{OA}\left(d^{2}, 5, d, 2\right)$ exists. (3) If $d \notin\{2,3,4,6,10$, $14,18,22\}$, then an $\mathrm{OA}\left(d^{2}, 6, d, 2\right)$ exists. (4) If $d \notin\{2,3,4,5,6,10$, $14,15,18,20,22,26,30,34,38,46,60,62\}=E$, then an $\mathrm{OA}\left(d^{2}, 7, d\right.$, 2) exists. (5) If $d \notin E \cup\{12,21,28,33,35,39,42,44,51,52,54$, $58,66,68,74\}$, then an $\mathrm{OA}\left(d^{2}, 8, d, 2\right)$ exists.

Lemma 1.11. ${ }^{2}$ If a $k$-uniform state of $N$ qudits exists, then $k \leq \frac{N}{2}$.

Lemma 1.12. (1) An OA $(r, N, d, k)$ is irredundant if and only if its minimal distance is greater than $k$. An $\operatorname{IrOA}(r, N, d, k)$ is an $\operatorname{IrOA}\left(r, N^{\prime}\right.$, $\left.d, k^{\prime}\right)$ for $N-k+k^{\prime} \leq N^{\prime} \leq N$ with $1 \leq k^{\prime}<k$. (2) An OA $(r, 2 k, d, k)$ is irredundant if and only if $r=d^{k}$. (3) An OA $\left(d^{k+1}, 2 k+1, d, k\right)$ is irredundant if and only if it is of strength $k+1$. (4) If an $\mathrm{OA}(r, 2 k+1$, $d, k)$ is irredundant, then $r \leq d^{k+1}$.

Lemma 1.13. Suppose $B_{r}$ is an $\mathrm{OA}(r, r-1,2,2)$. Then $L=\mathbf{( 2 )} \oplus\left[\mathbf{0}_{r}\right.$ $\left.B_{r}\right]$ is an $\mathrm{OA}(2 r, r, 2,3)$ with minimal distance $\frac{r}{2}$.

Lemma 1.14. An $\mathrm{OA}(r, N, d, k)$ with minimal distance $w \geq k+1$ implies that an $\operatorname{IrOA}\left(r, N^{\prime}, d, k\right)$ exists for $N-w+k+1 \leq N^{\prime} \leq N$.

Lemma 1.15. Let $\left\{A_{1}, A_{2}, \ldots, A_{u}\right\}$ be an orthogonal partition of strength 1 of $A=\mathrm{OA}\left(r^{\prime}, N^{\prime}, d, k^{\prime}\right)$, and $\left\{B_{1}, B_{2}, \ldots, B_{v}\right\}$ be an orthogonal partition of strength 1 of $B=\mathrm{OA}\left(r^{\prime \prime}, N^{\prime \prime}, d, k^{\prime \prime}\right)$ for $r^{\prime}=d u$, $r^{\prime \prime}=d v, u \leq v$ and $k^{\prime}, k^{\prime \prime} \geq 2$. Suppose $\operatorname{MD}(A)=w_{1}$ and $\operatorname{MD}(B)=w_{2}$. Let $h=$ I.c.m. $\{u, v\}$. If $k^{\prime}, k^{\prime \prime} \geq 3$, the matrix $M=$ $\left(\mathbf{1}_{\frac{h}{u}} \otimes\left(A_{[1,2, \ldots, u]}, d\right), \mathbf{1}_{\frac{h}{v}} \otimes\left(d, B_{[1,2, \ldots, v]}\right)\right)$ is an $\mathrm{OA}\left(d^{2} h, N^{\prime}+N^{\prime \prime}, d\right.$, 3). Otherwise, the matrix $M$ is an $\mathrm{OA}\left(d^{2} h, N^{\prime}+N^{\prime \prime}, d, 2\right)$, and

$\operatorname{MD}(M) \geq\left\{\begin{array}{l}\min \left\{w_{1}+w_{2}, N^{\prime}, N^{\prime \prime}\right\}, \text { if } u=v, \\ \min \left\{N^{\prime}, w_{2}\right\}, \text { if } u \mid v, u<v, \\ \min \left\{w_{1}, w_{2}\right\}, \text { otherwise. }\end{array}\right.$
Lemma 1.16. The minimal distance of an $\mathrm{OA}\left(d^{k}, N, d, k\right)$ is $N-k+1$ for $d \geq 2$ and $k \geq 1$.

Lemma 1.17. For any integer $d \geq 2$,. (1) If $d \notin\{2,6\}$, then an $\operatorname{IrOA}\left(d^{2}\right.$, $4, d, 2)$ exists. (2) If $d \notin\{2,3,6,10\}$, then an $\operatorname{IrOA}\left(d^{2}, N, d, 2\right)$ exists for $N=4,5$. (3) If $d \notin\{2,3,4,6,10,14,18,22\}$, then an $\operatorname{IrOA}\left(d^{2}, N, d, 2\right)$ exists for $4 \leq N \leq 6$. (4) If $d \notin\{2,3,4,5,6,10,14,15,18,20,22,26$, $30,34,38,46,60,62\}=E$, then an $\operatorname{IrOA}\left(d^{2}, N, d, 2\right)$ exists for $4 \leq N \leq$ 7. (5) If $d \notin E \cup\{12,21,28,33,35,39,42,44,51,52,54,58,66$, $68,74\}$, then an $\operatorname{IrOA}\left(d^{2}, N, d, 2\right)$ exists for $4 \leq N \leq 8$.

Lemma 1.18. Let $\left\{A_{1}, A_{2}, \ldots, A_{u}\right\}$ be an orthogonal partition of strength 1 of $\mathrm{OA}\left(r^{\prime}, N^{\prime}, d, k^{\prime}\right)$, and $\left\{B_{1}, B_{2}, \ldots, B_{u}\right\}$ be an orthogonal partition of strength 0 of $\mathrm{OA}\left(u, N^{\prime \prime}, d, k^{\prime \prime}\right)$ for $r^{\prime}=d u$ and $k^{\prime} \geq 2, k^{\prime \prime} \geq$ 2. Suppose the minimal distances of the two arrays $\mathrm{OA}\left(r^{\prime}, N^{\prime}, d, k^{\prime}\right)$ and $\mathrm{OA}\left(u, N^{\prime \prime}, d, k^{\prime \prime}\right)$ are $w_{1}$ and $w_{2}$, respectively. Then the matrix $M$ $=\left(\left(A_{[1,2, \ldots, u]}, 1\right),\left(d, B_{[1,2, \ldots, u]}\right)\right)$ is an $\mathrm{OA}\left(r^{\prime}, N^{\prime}+N^{\prime \prime}, d, 2\right)$ and its minimal distance satisfies $w \geq \min \left\{w_{1}+w_{2}, N^{\prime}\right\}$.

These lemmas will form the backbone of our main theorems and results. Especially, Lemmas 1.6, 1.15, and 1.18 play an essential role in finding infinite classes of uniform states. In the 'Discussion' section, we discuss the main results and some uniform states that could have fewer terms or qudits. In the "Methods" section, we describe the central idea of the construction method. We present some examples of 2 and 3-uniform states, summarize the constructed IrOAs, and present the proofs of some lemmas in the appendix (see Supplementary Information for a detailed description of the examples, IrOAs and proofs).

\section{RESULTS}

The main results are summarized in Table 1.

Very interestingly, not only can 2 and 3-uniform states of qubits be constructed using IrOAs, but the states of high-dimensional quantum systems (qudits) can also be obtained. We now address the open issue proposed by Goyeneche et al. ${ }^{2}$. There is a reciprocal link between $k$-uniform states of $N$ qudits and $((N, 1, k+$ 1)) $)_{d}$ QECCs. ${ }^{3}$ The newly constructed uniform states might be applied in quantum information protocols, quantum secret sharing, ${ }^{39}$ and holographic codes.

Using difference schemes and orthogonal partitions, we propose a recursive construction method for OAs. By exploring the Hamming distances of OAs, especially newly constructed OAs, we explicitly construct a finite set of $\operatorname{IrOA}(r, N, d, k)$ for $n \leq N \leq m$. The recursive nature of the method allows us to generate infinite classes of IrOAs (see Table 1). Relying on Connection 1.1, we prove the existence of the uniform states in Table 1. Some examples are presented.

First, we describe the construction of 3-uniform states from IrOAs.

Table 1. Existence of $k$-uniform states of $N$ qudits $(d \geq 2)$ and $\operatorname{IrOA}(r, N, d, k)$

\begin{tabular}{lllll}
\hline$k$ & $d$ & $k$-uniform states of $N$ qudits & $\operatorname{IrOA}(r, N, d, k)$ & Ref. \\
\hline$k=3$ & $d=2$ & $N \geq 8$ & $N=8$ and $N \geq 10$ & Th. 2.1 $N=9$ (Supplementary Ex. 4$)$ \\
& $d=3$ & $N \geq 8$ & $N \geq 8$ & Th. 2.4 \\
& $d=4,5$ & $N=6$ and $N \geq 8$ & $N=6$ and $N \geq 8$ & Th. 2.6 \\
& $d>6$ is a prime power & $N \geq 6$ & $N \geq 6$ & Th. 2.7 \\
& $d \geq 6$ is not a prime power & $N=8$ and $N \geq 12$ & $N=8$ and $N \geq 12$ & Th. 2.8 \\
& $d=2$ & $N \geq 5$ & $N \geq 6$ & Th. 2.11 \\
& $d=6$ & $N \geq 5$ & $N \geq 5$ & Th. 2.14 \\
& $d=10$ & $N=4$ and $N \geq 6$ & $N=4$ and $N \geq 6$ & Th. 2.16 \\
& $d \geq 2, d \notin\{2,6,10\}$ & $N \geq 4$ & $N \geq 4$ & Ths. 2.12, $2.13,2.15 \& 2.17$
\end{tabular}


Construction of 3-uniform states of $N \geq 8$ qubits

By using the minimal distance and other properties of OAs, we can obtain an $\operatorname{IrOA}(r, N, 2,3)$ and 3-uniform states of $N$ qubits for $N=8$ and $N \geq 10$. We also find a 3-uniform state of 9 qubits by adding some unimodular complex numbers in Appendix A (see Supplementary Example 4). Hence, we have 3-uniform states of $N$ qubits for every $N \geq 8$.

Theorem 2.1. There exists an $\operatorname{IrOA}(r, N, 2,3)$ for $N=8$ and every $N \geq$ 10 and 3-uniform states of $N$ qubits for every $N \geq 8$. Proof. Suppose that $n \geq 3$. It follows from Lemma 1.4 and Lemma 1.13 that an OA $\left(2^{n+1}, 2^{n}, 2,3\right)$ exists. From Lemmas 1.13 and 1.14 we can construct an $\operatorname{IrOA}\left(2^{n+1}, N_{n}, 2,3\right)$ for $2^{n-1}+4 \leq N_{n} \leq 2^{n}$. Obviously, $2^{n}+4 \leq$ $N_{n+1} \leq 2^{n+1}$. Therefore we can obtain $N_{3}=8,12 \leq N_{4} \leq 16,20 \leq$ $N_{5} \leq 32$, etc. On the other hand, starting from the $\mathrm{OA}(12,11,2,2)$ and using Lemma 1.5 , we can obtain an $\mathrm{OA}\left(2^{n-1} \times 3,2^{n-1} \times 3-1\right.$, $2,2)$ for $n \geq 3$. Then an $\operatorname{OA}\left(2^{n} \times 3,2^{n-1} \times 3,2,3\right)$ exists with the form as in Lemma 1.13. From Lemmas 1.13 and 1.14 we can also construct an $\operatorname{IrOA}\left(2^{n} \times 3, N_{n}^{\prime}, 2,3\right)$ for $2^{n-2} \times 3+4 \leq N_{n}^{\prime} \leq 2^{n-1} \times 3$. Thus we can respectively obtain $10 \leq N_{3}^{\prime} \leq 12$, $16 \leq N_{4}^{\prime} \leq 24$, $28 \leq N_{5}^{\prime} \leq 48$, and so on. And it can be easily seen that $2^{n-2} \times 3+$ $4 \leq 2^{n}$ and $2^{n}+4<2^{n-1} \times 3$ when $n \geq 4$. Therefore, we can obtain an $\operatorname{IrOA}(r, N, 2,3)$ for $N=8$ and $N \geq 10$. Every row of the $\operatorname{IrOA}(r, N$, $2,3)$ is put in kets, summed, and normalized to produce the desired 3-uniform states of $N$ qubits for $N=8$ and $N \geq 10$. The 3uniform state of $N=9$ qubits will be presented in Appendix A (see Supplementary Example 4).

This theorem suggests there is an infinite class of entangled states of $\mathrm{N}$-party systems such that, tracing out any $\mathrm{N}-3$ subsystems, the remaining three subsystems have associated a maximally mixed state. Therefore, this plays an important role in completely settling the question on the existence of 3-uniform states of $N$ qubits. It is also of vital importance to generalize qubits to high-dimensional quantum systems (qudits) because of the many advantages they offer over qubit-based applications.

Construction of 3-uniform states of $N \geq 8$ qutrits $(d=3)$

By studying the minimal distance of the symmetrical OAs constructed from the orthogonal partition methods in, ${ }^{32}$ we can obtain an $\operatorname{IrOA}(r, N, 3,3)$ and 3-uniform states of $N$ qutrits for every $N \geq 12$. By constructing $\mathrm{OA}(81,10,3,3)$ and $\operatorname{OA}(243,11,3,3)$ and computing their minimal distances, we have an $\operatorname{IrOA}(r, N, 3,3)$ for $N=8,9,10,11$. Therefore, an $\operatorname{IrOA}(r, N, 3,3)$ and 3-uniform states of $N$ qutrits can be obtained for every $N \geq 8$.

Theorem 2.2. There exists an $\operatorname{IrOA}\left(3^{n}, N_{n}, 3,3\right)$ and 3-uniform states of $N_{n}$ qutrits for $9 \cdot 2^{n-5}+4 \leq N_{n} \leq 9 \cdot 2^{n-4}$ with $n \geq 5$. Proof. First by using mathematical induction, we prove that there exists an $O A$ $\left(3^{n}, 9 \cdot 2^{n-4}, 3,3\right)$ having minimal distance $9 \cdot 2^{n-5}$ and an orthogonal partition $\left\{B_{1}, B_{2}, \ldots, B_{3^{n-1}}\right\}$ of strength 1 for any integer $n \geq 5$. When $n=5$, we can obtain an $\mathrm{OA}(81,9,3,3)=$ $D_{3}(27,9,3) \oplus(3)$ with minimal distance 5 from the following difference scheme:

$$
D_{3}(27,9,3)=\left(\begin{array}{c}
\mathbf{p}_{1} \\
\mathbf{p}_{2} \\
\vdots \\
\mathbf{p}_{27}
\end{array}\right)=\left(\begin{array}{c}
000000000000000000000000000 \\
012201120201120012120012201 \\
021210102102021210210102021 \\
012201120012201120012201120 \\
012012012120120120201201201 \\
000222111111000222222111000 \\
000000000111111111222222222 \\
000111222000111222000111222 \\
012012012012012012012012012
\end{array}\right)^{T} .
$$

Since $A_{i}=\mathrm{P}_{i} \oplus(3)$ is an $\mathrm{OA}$ of strength 1 for each $1 \leq i \leq 27,\left\{A_{1}\right.$, $\left.A_{2}, \ldots, A_{27}\right\}$ is an orthogonal partition of strength 1 of the $\mathrm{OA}(81,9$,
3, 3). By Lemma 1.6, we can obtain an

$\mathrm{OA}(243,18,3,3)=\left(\left(A_{[1,2, \ldots, 27]}, 3\right),\left(3, A_{[1,2, \ldots, 27]}\right)\right)$.

It follows from Lemma 1.15 that $\operatorname{MD}(\operatorname{OA}(243,18,3,3))=9$. From Lemma 1.7, there exists an orthogonal partition $\left\{A_{i 1}, A_{i 2}, A_{i 3}\right\}$ of strength 1 of $\mathrm{OA}\left(A_{i} \otimes \mathbf{1}_{3}, \mathbf{1}_{3} \otimes A_{i}\right)$ for each $1 \leq i \leq 27$. Then there also exists an orthogonal partition $\left\{B_{1}, B_{2}, \ldots, B_{81}\right\}$ of strength 1 of the $\mathrm{OA}(243,18,3,3)$. Assume that when $n=m$ there exists an $\mathrm{OA}$ $\left(3^{m}, 9 \cdot 2^{m-4}, 3,3\right)$ with minimal distance $9 \cdot 2^{m-5}$ and an orthogonal partition $\left\{C_{1}, C_{2}, \ldots, C_{3^{m-1}}\right\}$ of strength 1 . When $n=$ $m+1$, by induction and Lemmas 1.6 and 1.15, we can obtain an

$\mathrm{OA}\left(3^{m+1}, 9 \cdot 2^{m-3}, 3,3\right)=\left(\left(C_{\left[1,2, \ldots, 3^{m-1}\right]}, 3\right),\left(3, C_{\left[1,2, \ldots, 3^{m-1}\right]}\right)\right)$

whose minimal distance is $9 \cdot 2^{m-4}$. From Lemma 1.7, an orthogonal partition $\left\{C_{i 1}, C_{i 2}, C_{i 3}\right\}$ of strength 1 of $\mathrm{OA}\left(C_{i} \otimes \mathbf{1}_{3}\right.$, $\left.\mathbf{1}_{3} \otimes C_{i}\right)$ exists for $1 \leq i \leq 3^{m-1}$, so does an orthogonal partition $\left\{D_{1}, D_{2}, \ldots, D_{3^{m}}\right\}$ of strength 1 of the $\mathrm{OA}\left(3^{m+1}, 9 \cdot 2^{m-3}, 3,3\right)$. From Lemma 1.14 , we can obtain an $\operatorname{IrOA}\left(3^{n}, N_{n}, 3,3\right)$ for $9 \cdot 2^{n-5}$ $+4 \leq N_{n} \leq 9 \cdot 2^{n-4}$ with $n \geq 5$. The desired 3-uniform states follow from Connection 1.1.

For example, using Theorem 2.2 we can obtain an $\operatorname{IrOA}(243, N$, $3,3)$ for $13 \leq N \leq 18$ from the $\mathrm{OA}(243,18,3,3)$, an $\operatorname{IrOA}(729, N, 3,3)$ for $22 \leq N \leq 36$ from an $\mathrm{OA}(729,36,3,3)$ and an $\operatorname{IrOA}(2187, N, 3,3)$ for $40 \leq N \leq 72$ from an $\mathrm{OA}(2187,72,3,3)$, etc.

Theorem 2.3. There exists an $\operatorname{IrOA}\left(3^{n}, N_{n}^{\prime}, 3,3\right)$ and 3-uniform states of $N_{n}^{\prime}$ qutrits for $9 \cdot 2^{n-6}+8 \leq N_{n}^{\prime} \leq 9 \cdot 2^{n-5}+4$ with $n \geq 6$. Proof. We can obtain an $\mathrm{OA}(27,4,3,3)=D_{3}(9,4,3) \oplus$ (3) with an orthogonal partition $\left\{A_{1}, A_{2}, \ldots, A_{9}\right\}$ of strength 1. From Theorem 2.2 , there exists an $\operatorname{IrOA}\left(3^{n-1}, N_{n-1}, 3,3\right)$ for $9 \cdot 2^{n-6}+4 \leq N_{n-1} \leq$ $9 \cdot 2^{n-5}$ and its an orthogonal partition $\left\{B_{1}, B_{2}, \ldots, B_{3^{n-2}}\right\}$ of strength 1 for $n \geq 6$. As $9<3^{n-2}$ and $9 \mid 3^{n-2}$, from Lemma 1.6 we can obtain an matrix $M=\left(\mathbf{1}_{3^{n-4}} \otimes\left(A_{[1,2, \ldots, 9]}, 3\right),\left(3, B_{\left[1,2, \ldots, 3^{n-2}\right]}\right)\right)$. It is an $\mathrm{OA}\left(3^{n}, N_{n}^{\prime}, 3,3\right)$ since $N_{n^{\prime}}=N_{n-1}+4$. By the case of $u \mid v, u<$ $v$ in Lemma 1.15, we have $\operatorname{MD}\left(\mathrm{OA}\left(3^{n}, N_{n}^{\prime}, 3,3\right)\right) \geq \min \{4, \mathrm{MD}(\operatorname{IrOA}$ $\left.\left.\left(3^{n-1}, N_{n-1}, 3,3\right)\right)\right\}=4$. Hence it is an $\operatorname{IrOA}\left(3^{n}, N_{n}^{\prime}, 3,3\right)$ for $9 \cdot 2^{n-6}$ $+8 \leq N_{n^{\prime}} \leq 9 \cdot 2^{n-5}+4$.

From Theorem 2.3, we can obtain an $\operatorname{IrOA}(729, N, 3,3)$ for $17 \leq$ $N \leq 22$. We can also obtain an $\operatorname{IrOA}(2187, N, 3,3)$ for $26 \leq N \leq$ 40 , etc.

Theorem 2.4. There exists an $\operatorname{IrOA}(r, N, 3,3)$ and 3-uniform states of $N$ qutrits for every $N \geq 8$. Proof. By Theorems 2.2 and 2.3, we have an $\operatorname{IrOA}\left(3^{n}, N_{n}, 3,3\right)$ for $9 \cdot 2^{n-6}+8 \leq N_{n} \leq 9 \cdot 2^{n-4}$ with $n \geq 6$. Since $9 \cdot 2^{n-5}+8 \leq N_{n+1} \leq 9 \cdot 2^{n-3}$ and $9 \cdot 2^{n-5}+8<9 \cdot 2^{n-4}$ for $n \geq 6$, we can obtain an $\operatorname{IrOA}(r, N, 3,3)$ for $N \geq 17$. Taking $n=5$ in Theorem 2.2, we can have an $\operatorname{IrOA}\left(3^{5}, N, 3,3\right)$ for $13 \leq N \leq 18$. By removing a column from the $\mathrm{OA}(81,9,3,3)$ in Theorem 2.2 , we can obtain an $\mathrm{OA}(81,8,3,3)$ with minimal distance $w_{2} \geq 4$. By using Lemmas 1.6 and 1.7 and the case of $u \mid v, u<v$ in Lemma 1.15, from the two arrays $\operatorname{OA}(27,4,3,3)$ and $\operatorname{OA}(81,8,3,3)$ we can construct an $\mathrm{OA}(243,12,3,3)$ with minimal distance $w \geq \min \{4$, $\left.w_{2}\right\}=4$. So we can obtain an $\operatorname{IrOA}(243,12,3,3)$. Taking $C=\left(\begin{array}{l}10000111111 \\ 01011011011 \\ 00111111111 \\ 01000111222 \\ 10012012012\end{array}\right)$, by the method in $^{40}$ we have an OA

$(243,11,3,3)$ with minimal distance 4 . So we can get an $\operatorname{IrOA}(243$, $11,3,3)$. From the construction of an $\mathrm{OA}\left(d^{4}, d^{2}+1, d, 3\right)$ in, ${ }^{12}$ we obtain an $\operatorname{OA}(81,10,3,3)$ whose minimal distance is 6 . So we can obtain an $\operatorname{IrOA}(81, N, 3,3)$ for $N=8,9,10$. The desired conclusion follows from Connection 1.1. 
Interestingly, this theorem explicitly constructs a class of 3uniform states that do not contain any terms with complex phase weights. And every subsystem of $N^{\prime} \leq 3$ qutrits contains the same physical information. Orthogonal partitions of OAs play a key role in the proof of this theorem. Starting from a judiciously selected difference scheme and using a similar method, we obtain a general result.

Theorem 2.5. If there exists a $D_{k}(r, c, d)$ with $c \geq k+1$ for $k \geq 2$ such that the minimal distance of $L=\mathrm{OA}(r d, c, d, k)=D_{k}(r, c, d) \oplus(\mathbf{d})$ is at least $\left[\frac{c}{2}\right]$, then we have an $\operatorname{IrOA}(m, N, d, 3)$ and 3-uniform states of $N$ qudits for $c+4 \leq N \leq 2 c$ and every $N \geq c+8$, and we also have an $\operatorname{IrOA}(m, N, d, 2)$ and 2-uniform states of $N$ qudits for $c+3 \leq N \leq$ $2 c$ and every $N \geq c+6$. Proof. When $k \geq 3$, by using Lemmas 1.6 and 1.7 and the case of $u=v$ in Lemma 1.15, starting from $L$ we can recursively construct a class of OAs $\mathrm{OA}\left(r d^{n+1}, 2^{n} c, d, 3\right)$ with minimal distance $2^{n-1} c$ for any integer $n \geq 1$. Then we can obtain an $\operatorname{IrOA}\left(r d^{n+1}, N_{n}^{\prime}, d, 3\right)$ for $2^{n-1} c+4 \leq N_{n}^{\prime} \leq 2^{n} c$. When $n=1$, we have $\operatorname{IrOA}\left(r d^{2}, N, d, 3\right)$ for $c+4 \leq N \leq 2 c$. By using Lemmas 1.6 and 1.7 and the case of $u \mid v, u<v$ in Lemma 1.15, from the arrays OA $(r d$, $4, d, k)=D_{k}(r, 4, d) \oplus$ (d) and $\operatorname{IrOA}\left(r d^{n+1}, N_{n}^{\prime}, d, 3\right)$ we can construct $\mathrm{OA}\left(r d^{n+2}, N_{n+1}^{\prime \prime}, d, 3\right) \quad$ for $\quad 2^{n-1} c+8 \leq N_{n+1}^{\prime \prime} \leq 2^{n} c+4 \quad$ with $\operatorname{MD}\left(\mathrm{OA}\left(r d^{n+2}, N_{n+1}^{\prime \prime}, d, 3\right)\right) \geq \min \left\{4, \operatorname{MD}\left(\operatorname{IrOA}\left(r d^{n+1}, N_{n}^{\prime}, d, 3\right)\right)\right\}=4$ since $N_{n+1}^{\prime \prime}=N_{n}^{\prime}+4$. They are IrOAs. Hence an OA $\left(r d^{n+1}, N_{n}^{\prime \prime}, d, 3\right)$ exists for $2^{n-2} c+8 \leq N_{n}^{\prime \prime} \leq 2^{n-1} c+4$ with $n \geq 2$. Then an $\mathrm{OA}\left(r d^{n}\right.$ $\left.{ }^{+1}, N_{n}, d, 3\right)$ exists for $2^{n-2} c+8 \leq N_{n} \leq 2^{n} c$ with $n \geq 2$. Since $2^{n-1} c$ $+8 \leq N_{n+1} \leq 2^{n+1} c$ and $2^{n} c \geq 2^{n-1} c+8$, we can obtain an $\operatorname{IrOA}(m$, $N, d, 3)$ for any integer $N \geq c+8$. Similarly, when $k \geq 2$, we also have an $\operatorname{IrOA}(m, N, d, 2)$ and 2-uniform states of $N$ qudits for $c+$ $3 \leq N \leq 2 c$ every $N \geq c+6$.

Based on Theorem 2.5, we further consider the cases of qudits $(d \geq 4)$.

Construction of 3-uniform states of $N=6$ or $N \geq 8$ ququarts and ququints $(d=4,5)$

By analyzing the minimal distances of the symmetrical OAs constructed from Theorem 2.5, we obtain two infinite classes of $\operatorname{IrOA}(r, N, 4,3)$ and $\operatorname{IrOA}(r, N, 5,3)$ and 3-uniform states of $N$ ququarts and ququints for $N=6$ and every $N \geq 8$.

Theorem 2.6. There exists an $\operatorname{IrOA}(r, N, d, 3)$ for $d=4,5$ and 3-uniform states of $N$ ququarts and ququints for $N=6$ and every $N \geq$ 8. Proof. Consider the case $d=4$. By Lemma 1.16 we can obtain the $\mathrm{OA}(64,6,4,3)=D_{3}(16,6,4) \oplus(4)$ with minimal distance 4 , where

$D_{3}(16,6,4)=\left(\begin{array}{l}0000000000000000 \\ 0123230132101032 \\ 0231132020133102 \\ 0231201331021320 \\ 0000111122223333 \\ 0123012301230123\end{array}\right)^{T}$.

Then an $\operatorname{IrOA}(64, N, 4,3)$ exists for $N=6$. It follows from Theorem 2.5 that starting from the $D_{3}(16,6,4)$ we have an $\operatorname{IrOA}(r, N, 4,3)$ for $10 \leq N \leq 12$ and every $N \geq 14$. Now we prove the existence of an $\operatorname{IrOA}(r, N, 4,3)$ for $N=8,9$, 13. From the $\mathrm{OA}(64,6,4,3)$ we can easily have two arrays $\operatorname{OA}(64,5,4,3)$ and $\operatorname{OA}(64,4,4,3)$ with minimal distances 3 and 2 . We proceed our proof by using Lemmas 1.6, 1.7 and 1.15. From the $\operatorname{OA}(64,4,4,3)$ we can obtain $\mathrm{OA}(256,8,4,3)$. From the arrays $\mathrm{OA}(64,4,4,3)$ and $\mathrm{OA}(64,5,4,3)$ we can obtain $\mathrm{OA}(256,9,4,3)$. Moreover, from the two arrays $\mathrm{OA}$ $(64,5,4,3)$ and $\mathrm{OA}(256,8,4,3)$ we can construct an $\mathrm{OA}(1024,13$, $4,3)$. They all have minimal distances $\geq 4$. Then three arrays IrOA $(256,8,4,3), \operatorname{IrOA}(256,9,4,3)$ and $\operatorname{IrOA}(1024,13,4,3)$ exist. Therefore, we have an $\operatorname{IrOA}(r, N, 4,3)$ and 3-uniform states of $N$ ququarts for $N=6$ and every $N \geq 8$. Similarly, when $d=5$, from the $D_{3}(25,6,5)$ in $^{35}$ we also have an $\operatorname{IrOA}(r, N, 5,3)$ and 3 -uniform states of $N$ ququints for $N=6$ and every $N \geq 8$.

Construction of 3-uniform states of $N$ qudits with $d \geq 6$ levels

Starting from the difference schemes $D_{3}\left(d^{2}, d, d\right)$ and $D_{3}\left(d^{2}, 4, d\right)$ respectively and exploring the minimal distances of $O A s$, we will construct an $\operatorname{IrOA}(r, N, d, 3)$ and the 3-uniform states of $N$ qudits for every $N \geq 6$ with any prime power $d>6$ levels, and an $\operatorname{IrOA}(r, N, d$, 3 ) and the 3-uniform states of $N$ qudits for $N=8$ and every $N \geq 12$ with any non-prime-power $d \geq 6$ levels.

Theorem 2.7. For any prime power $d>6$, there exists an $\operatorname{IrOA}(r, N, d$, 3) and 3-uniform states of $N$ qudits for every $N \geq 6$. Proof. By Lemmas 1.4 and 1.16 , we can get an $\mathrm{OA}\left(d^{3}, d+1, d, 3\right)$ with minimal distance $d-1$, so we have an $\operatorname{IrOA}\left(d^{3}, N, d, 3\right)$ for $6 \leq N \leq d$ +1 from Lemma 1.14. It follows from Lemmas 1.3 and 1.16 that a difference scheme $D_{3}\left(d^{2}, d, d\right)$ exists and the minimal distance of $\mathrm{OA}\left(d^{3}, d, d, 3\right)=D_{3}\left(d^{2}, d, d\right) \oplus(\mathbf{d})$ is $d-2$. Then an $\mathrm{OA}\left(d^{3}, N_{3}, d, 3\right)$ exists with minimal distance $\geq 2$ for $4 \leq N_{3} \leq d$. From the OA $\left(d^{3}, N_{3}\right.$, $d, 3)\left(4 \leq N_{3} \leq d\right)$ and Lemmas 1.6 and 1.7 and the case of $u=v$ in Lemma 1.15, we can construct an $\mathrm{OA}\left(d^{4}, N, d, 3\right)$ with minimal distance $d \geq\{2+2,4,4\}=4$. They are all $\operatorname{IrOA}\left(d^{4}, N, d, 3\right)$ for $8 \leq$ $N \leq 2 d$. By Theorem 2.5 and from the $D_{3}\left(d^{2}, d, d\right)$, we can obtain an $\operatorname{IrOA}(r, N, d, 3)$ for every $N \geq d+8$. Since $7<d+1$ and $d+7 \leq 2 d$, we can obtain an $\operatorname{IrOA}(r, N, d, 3)$ for every $N \geq 6$. From Connection 1.1 , we can obtain an $\operatorname{IrOA}(r, N, d, 3)$ and 3-uniform states of $N$ qudits for every $N \geq 6$.

Using similar arguments, we can construct 3-uniform states with any non-prime-power $d \geq 6$ levels.

Theorem 2.8. For any non-prime-power $d \geq 6$, there exists an $\operatorname{IrOA}(r$, $N, d, 3)$ and 3-uniform states of $N$ qudits for $N=8$ and every $N \geq 12$. Proof. From Lemma 1.3, a difference scheme $D_{3}\left(d^{2}, 4, d\right)$ exists for any $d \geq 2$. It follows from Lemma 1.16 that the minimal distance of $\mathrm{OA}\left(d^{3}, 4, d, 3\right)=D_{3}\left(d^{2}, 4, d\right) \oplus(\mathbf{d})$ is 2 . Therefore, we have an IrOA $(r, N, d, 3)$ for $N=8$ and every $N \geq 12$ by Theorem 2.5. By Connection 1.1, we can obtain 3-uniform states of $N$ qudits for $N$ $=8$ and every $N \geq 12$.

The existence of maximally entangled states lies at the intersection of quantum theory and discrete mathematics, and determining their mathematical structure is extremely technical. The characterization and classification of such entanglement have now become issues of great interest. The constructed states with higher dimensions offer an increase in the channel capacity for quantum communication, and provide a higher error rate tolerance and enhanced security in quantum key distribution. ${ }^{41}$ High-dimensional entanglement offers great potential for applications in quantum information, particularly in quantum communications.

However, the states of higher dimensions are significantly different from the trivial extension of those of lower dimensions. A key problem in the construction of 3-uniform states of a nonprime-power dimension is the shortage of proper mathematical tools. For example, in non-prime-power dimensions the Galois fields do not exist. Therefore, studying these states is even more challenging than in the case of prime-power dimensions. In practice, the available experimental data are usually quite limited, such that devising effective ways for characterizing highdimensional entanglement is very challenging. Interestingly, the states of any dimensions can be consistently obtained using the proposed methods.

It is interesting that 3-uniform states are a natural extension of 1-uniform states. They are useful for increasing the order of density coding information rate from $d^{1}$ to $d^{3}$. ${ }^{20}$ Moreover, a 3-uniform state is also 2-uniform. 
We continue to use our method for 2-uniform states.

New results of construction of 2-uniform states of $N \geq 5$ qubits

For $N \geq 6$, there exist 2-uniform states of $N$ qubits that can be constructed from two infinite classes of Hadamard matrices based on the Hadamard matrices $H_{2}$ and $H_{12}$ of orders 2 and $12 .{ }^{2}$ However, we simplify the construction of these 2-uniform states using only the known class of orthogonal arrays $\operatorname{OA}\left(2^{n}, 2^{n}-1,2,2\right)$ with $n \geq 3$.

Theorem 2.9. A saturated orthogonal array $\mathrm{OA}(r, r-1,2,2)$ for $r \geq 8$ implies the existence of an $\operatorname{IrOA}(r, N, 2,2)$ and 2-uniform states of $N$ qubits for $\frac{r}{2}+2 \leq N \leq r-1$. Proof. It follows from Lemma 1.2 that the $\mathrm{OA}(r, r-1,2,2)$ has constant Hamming distance $\frac{r}{2}$. Then we have an $\operatorname{IrOA}(r, N, 2,2)$ for $\frac{r}{2}+2 \leq N \leq r-1$ from Lemma 1.14. By Connection 1.1, we can obtain 2-uniform states of $N$ qubits for $\frac{r}{2}+2 \leq N \leq r-1$.

Example 2.1. By Theorem 2.9, we can obtain an $\operatorname{IrOA}(8, N, 2,2)$ and 2-uniform states of $N$ qubits for $6 \leq N \leq 7$, an $\operatorname{IrOA}(12, N, 2,2)$ and 2uniform states of $N$ qubits for $8 \leq N \leq 11$ and an $\operatorname{IrOA}(20, N, 2,2)$ and 2-uniform states of $N$ qubits for $12 \leq N \leq 19$ from $O A(8,7,2,2)$, OA $(12,11,2,2)$ and $\mathrm{OA}(20,19,2,2)$, respectively.

Theorem 2.10. If $B_{\frac{r}{2}}$ is an $\mathrm{OA}\left(\frac{r}{2}, \frac{r}{2}-1,2,2\right)$ for $r \geq 16$ and $8 \mid r$, then the array $L=\left[B_{\frac{r}{2}} \oplus \mathbf{0}_{2},\left(\mathbf{0}_{\frac{r}{2}}, B_{\frac{r}{2}}\right) \oplus(\mathbf{2})\right]$ allows us to obtain an $\operatorname{IrOA}(r$, $\left.N_{r}, 2,2\right)$ for $\frac{r}{4}+3 \leq N_{r} \leq r-1$. Proof. If $B_{\frac{r}{2}}$ is an $O A$, then $L$ is an OA $(r, r-1,2,2)$ by Lemma 1.5. Let $L_{1}=B_{\frac{r}{2}} \oplus \mathbf{0}_{2}, L_{2}=\left(\mathbf{0}_{\frac{r}{2}}, B_{\frac{r}{2}}\right) \oplus(\mathbf{2})$. It follows from Lemma 1.2 that the Hamming distances $H D, H D_{1}$, $\mathrm{HD}_{2}$ of $L, L_{1}, L_{2}$ statisfy $\mathrm{HD}=\frac{r}{2}, \mathrm{HD}_{1} \leq \frac{r}{4}, \mathrm{HD}_{2} \geq \frac{r}{4}$. Consequently, we can obtain an $\operatorname{IrOA}\left(r, N_{r}, 2,2\right)$ for $\frac{r}{4}+3 \leq N_{r} \leq r-1$ by respectively deleting $j\left(0 \leq j \leq \frac{3}{4} r-4\right)$ columns from the $L$. In fact, if $0 \leq j \leq \frac{r}{2}-1$, by deleting arbitrary $j$ columns in $L_{1}$, we can obtain an IrOA $\left(r, N_{r}, 2,2\right)$ for $\frac{r}{2} \leq N_{r} \leq r-1$. If $\frac{r}{2}-1<j \leq \frac{3}{4} r-4$, by deleting all columns in $L_{1}$ and arbitrary $j-\left(\frac{r}{2}-1\right)$ columns in $L_{2}$, we can obtain an $\operatorname{IrOA}\left(r, N_{r}, 2,2\right)$ for $\frac{r}{4}+3 \leq N_{r}<\frac{r}{2}$. We complete the proof.

Example 2.2. By Theorem 2.9 and an $\mathrm{OA}(24,23,2,2)$, we can obtain an $\operatorname{IrOA}(24, N, 2,2)$ and 2-uniform states of $N$ qubits for $14 \leq N \leq 23$. However, from the $\mathrm{OA}(24,23,2,2)$ satisfying the condition in Theorem 2.10 we can obtain more $\operatorname{IrOA}(24, N, 2,2)$ with $9 \leq N \leq 13$ than from Theorem 2.9. Thus we have $9 \leq N \leq 23$. Hence more 2uniform states can be obtained.

Theorem 2.11. An $\operatorname{IrOA}(r, N, 2,2)$ for $N \geq 6$ can be obtained from a known class of $\mathrm{OA}\left(2^{n}, 2^{n}-1,2,2\right)$ with $n \geq 3$ and 2-uniform states of $N$ qubits exist for every $N \geq 5$. Proof. In Theorem 2.10, let $B_{2^{n}}=$ $\mathrm{OA}\left(2^{n}, 2^{n}-1,2,2\right)$ which exists for every $n \geq 3$ from Lemma 1.5. Then we can obtain an $\operatorname{IrOA}\left(2^{n+1}, N_{2^{n+1}}, 2,2\right)$ for $2^{n-1}+3 \leq N_{2^{n+1}} \leq 2^{n+1}-1$, which does not include the Hadamard conjecture. So we have $2^{n}+3 \leq N_{2^{n+2}} \leq 2^{n+2}-1$. And it can be easily proved that $2^{n+1}-1>2^{n}+3$ when $n \geq 3$. Then we can get an $\operatorname{IrOA}(r, N, 2,2)$ for every $N \geq 7$. In particular, when $n=3$, using the $\mathrm{OA}(8,7,2,2)$ again we have $\operatorname{IrOA}(8,6,2,2)$ by Theorem 2.9 Therefore, there exists an $\operatorname{IrOA}(r, N, 2,2)$ for every $N \geq 6$. On the other hand, we have a 2-uniform state $\left|\Psi_{5}\right\rangle$ of $N=5$ qubits as follows: $\left|\Psi_{5}\right\rangle=|11111\rangle+|00110\rangle+|01100\rangle+|10101\rangle-|10000\rangle$ $+|01001\rangle-|00011\rangle+|11010\rangle$. Another expression of a 2-uniform state of five qubits can been found in. ${ }^{2}$. Hence, there exist 2uniform states of $N$ qubits for $N \geq 5$. We finish the proof.

Example 2.3. By Theorem 2.9, we can obtain an $\operatorname{IrOA}(16, N, 2,2)$ and 2-uniform states of $N$ qubits for $10 \leq N \leq 15$ from any $\mathrm{OA}(16,15,2$, 2). However, from the $\mathrm{OA}(16,15,2,2)$ satisfying the condition in Theorem 2.11 we can obtain more $\operatorname{IrOA}(16, N, 2,2)$ and 2-uniform states of $N$ qubits than for $7 \leq N \leq 15$ from Theorem 2.9.
Remark 1. Let $n=4$ in Theorem 2.11 Then we have IrOA(32, 31, 2, 2) for $11 \leq N_{32} \leq 31$. Further, we can obtain $9 \leq N_{32}{ }^{\prime} \leq 31$ by using the property of interaction columns (see Example 2.4).

Example 2.4. The existence of an $\operatorname{IrOA}(32, N, 2,2)$ and 2-uniform states of $N$ qubits for $9 \leq N \leq 31$.

By Theorem 2.9, we can obtain an $\operatorname{IrOA}(32, N, 2,2)$ and 2uniform states of $N$ qubits for $18 \leq N \leq 31$ from any $\mathrm{OA}(32,31,2,2)$. However, from the $\operatorname{OA}(32,31,2,2)$ satisfying the condition in Theorem 2.11 we can obtain $\operatorname{IrOA}(32, N, 2,2)$ and 2-uniform states of $N$ qubits for $11 \leq N \leq 31$. Let $B_{16}=O A(16,15,2,2)=\left(\mathbf{a}_{1}, \mathbf{b}_{1}, \mathbf{c}_{1}\right.$; $\left.\mathbf{a}_{2}, \mathbf{b}_{2}, \mathbf{c}_{2} ; \ldots ; \mathbf{a}_{5}, \mathbf{b}_{5}, \mathbf{c}_{5}\right)$ and $\mathrm{OA}(32,31,2,2)=\left[B_{16} \oplus \mathbf{0}_{2},\left(\mathbf{0}_{16}, B_{16}\right) \oplus\right.$ (2)]. Then the Hamming distance of the OA $\left[\mathbf{0}_{16}, B_{16}\right] \oplus$ (2) is greater than or equal to 8 . And the $\mathrm{OA}(16,15,2,2)$ has 6 columns $\left(\mathbf{a}_{4}, \mathbf{b}_{4}, \mathbf{c}_{4} ; \mathbf{a}_{5}, \mathbf{b}_{5}, \mathbf{c}_{5}\right)$ such that $\mathbf{c}_{i}$ is the interaction between $\mathbf{a}_{i}$ and $\mathbf{b}_{i}$ for $i=4,5$. The Hamming distance between the rows in the columns $\left(\mathbf{a}_{4}, \mathbf{b}_{4}, \mathbf{c}_{4} ; \mathbf{a}_{5}, \mathbf{b}_{5}, \mathbf{c}_{5}\right)$ is less than or equal to 4 . Then by respectively removing $\left(\mathbf{a}_{4}, \mathbf{b}_{4}, \mathbf{c}_{4} ; \mathbf{a}_{5}, \mathbf{b}_{5}, \mathbf{c}_{5}\right) \oplus(\mathbf{2})$ and $\left(\mathbf{c}_{3} ; \mathbf{a}_{4}, \mathbf{b}_{4}, \mathbf{c}_{4}\right.$; $\left.\mathbf{a}_{5}, \mathbf{b}_{5}, \mathbf{c}_{5}\right) \oplus(\mathbf{2})$ from $\left[\mathbf{0}_{16}, B_{16}\right] \oplus(\mathbf{2})$, one can obtain two arrays $\operatorname{IrOA}(32,10,2,2)$ and $\operatorname{IrOA}(32,9,2,2)$.

High-dimensional entanglement is different from two-level entanglement. Furthermore, general quantum states could be multilevel. They break the generic classical constraints and provide a new perspective for quantum mechanics.

Construction of 2-uniform states of $N \geq 4$ qutrits

We can derive an $\operatorname{IrOA}(r, N, 3,2)$ and 2-uniform states of $N$ qutrits for every $N \geq 4$ by the property and the minimal distances of OAs and difference schemes.

Theorem 2.12. There exists an $\operatorname{IrOA}(r, N, 3,2)$ and 2-uniform states of $N$ qutrits for every $N \geq 4$. Proof. We first prove that there exists an $\operatorname{IrOA}\left(3^{n+1}, N_{n}, 3,2\right)$ for $3^{n-1}+3 \leq N_{n} \leq \frac{3^{n+1}-1}{2}$ with $n \geq 1$. When $n$ $=1$, any $\operatorname{OA}(9,4,3,2)$ is an $\operatorname{IrOA}\left(3^{2}, N_{1}, 3,2\right)$ satisfying $4 \leq N_{1} \leq 4$. When $n \geq 2$, Lemmas 1.3 and 1.4 imply the existence of $\mathrm{OA}\left(3^{n}, \frac{3^{n}-1}{2}, 3,2\right)$ and $D\left(3^{n}, 3^{n}, 3\right)$. For a given $n$, let $B_{3^{n}}=\mathrm{OA}\left(3^{n}, \frac{3^{n}-1}{2}, 3,2\right), L_{1}=B_{3^{n}} \oplus \mathbf{0}_{3}$ and $L_{2}=D\left(3^{n}, 3^{n}, 3\right) \oplus$ (3). Then, $L=\left[L_{1}, L_{2}\right]$ is an $\mathrm{OA}\left(3^{n+1}, \frac{3^{n+1}-1}{2}, 3,2\right)$ from Lemma 1.5. It follows from Lemma 1.2 that the Hamming distances $H D, \mathrm{HD}_{1}$, and $\mathrm{HD}_{2}$ of $L, L_{1}$, and $L_{2}$ satisfy $\mathrm{HD}=3^{n}, \mathrm{HD}_{1} \leq 3^{n-1}\left(\mathrm{HD}_{1}=0,3^{n-1}\right)$, $\mathrm{HD}_{2} \geq 2 \cdot 3^{n-1}$. Consequently, we can obtain an $\operatorname{IrOA}\left(3^{n+1}, N_{n}, 3,2\right)$ for $3^{n-1}+3 \leq N_{n} \leq \frac{3^{n+1}-1}{2}$ by deleting $0 \leq j \leq \frac{7}{2}\left(3^{n-1}-1\right)$ columns from $L$. In fact, by deleting any $0 \leq j \leq \frac{3^{n}-1}{2}$ columns in $L_{1}$, we obtain an $\operatorname{IrOA}\left(3^{n+1}, N_{n}, 3,2\right)$ for $3^{n} \leq N_{n} \leq \frac{3^{n+1}-1}{2}$. For $\frac{3^{n}-1}{2}<j \leq \frac{7}{2}\left(3^{n-1}-1\right)$, deleting all the columns in $L_{1}$ and any $j-$ $\left(\frac{3^{n}-1}{2}\right)$ columns in $L_{2}$ gives $\operatorname{IrOA}\left(3^{n+1}, N_{n}, 3,2\right)$ for $3^{n-1}+3 \leq N_{n}<$ $3^{n}$. It can easily be shown that $3^{n-1}+3 \leq N_{n} \leq \frac{3^{n+1}-1}{2}$, $3^{n}+3 \leq N_{n+1} \leq \frac{3^{n+2}-1}{2}$, and $3^{n}+3<\frac{3^{n+1}-1}{2}$ for $n \geq 2$. When $n=$ $2,3,4, \ldots$, we can respectively obtain an $\operatorname{IrOA}\left(3^{n+1}, N_{n}, 3,2\right)$ for $6 \leq$ $N_{2} \leq 13,12 \leq N_{3} \leq 40,30 \leq N_{4} \leq 121$, and so on. Thus, we can obtain an $\operatorname{IrOA}(r, N, 3,2)$ for $N=4$ and every $N \geq 6$. When $N=5$, taking

$\mathrm{OA}(18,7,3,2)=\left(\mathbf{a}_{1}, \mathbf{a}_{2}, \ldots, \mathbf{a}_{7}\right)=\left(\begin{array}{c}012012012012012012 \\ 012012120201120201 \\ 012120012201201120 \\ 012201201012120120 \\ 012120201120012201 \\ 012201120120201012 \\ 000000111111222222\end{array}\right)^{T}$

we can obtain an $\operatorname{IrOA}(18,5,3,2)=\left(\mathbf{a}_{1}, \mathbf{a}_{2}, \ldots, \mathbf{a}_{5}\right)$ that leads to a 2 - 
uniform state of five qutrits. By Connection 1.1, we can obtain 2uniform states of $N$ qutrits for every $N \geq 4$.

For these states, every matrix in which the density is reduced to two qutrits is proportional to the identity $I_{3^{2}}$. This theorem settles the question of the 2-uniform states of $N$ qutrits. ${ }^{2}$ Note that the OAs $L$ in this theorem can be recursively constructed starting from $\mathrm{OA}(9,4,3,2)$.

Construction of 2-uniform states of $N \geq 4$ qudits with a prime power $d \geq 4$ levels

For a prime power $d \geq 4$, we can obtain an $\operatorname{IrOA}(r, N, d, 2)$ and 2uniform states of $N$ qudits for every $N \geq 4$ from the property and minimal distances of OAs and some special difference schemes.

Theorem 2.13. For a prime power $d \geq 4$, there exists an $\operatorname{IrOA}(r, N, d$, 2) and 2-uniform states of $N$ qudits for an arbitrary number of $N \geq 4$. Proof. For a prime power $d$ and an integer $n \geq 2$, Lemmas 1.3, 1.4, and 1.5 imply that there exists an $\mathrm{OA}$

$$
L=\mathrm{OA}\left(d^{n+1}, \frac{d^{n+1}-1}{d-1}, d, 2\right)=\left[B_{d^{n}} \oplus \mathbf{0}_{d}, D\left(d^{n}, d^{n}, d\right) \oplus(\mathbf{d})\right]
$$

where $B_{d^{n}}=\mathrm{OA}\left(d^{n}, \frac{d^{n}-1}{d-1}, d, 2\right)$. Let $L_{1}=B_{d^{n}} \oplus \mathbf{0}_{d}$ and $L_{2}=D\left(d^{n}\right.$, $\left.d^{n}, d\right) \oplus(\mathbf{d})$. Then, $L=\left[L_{1}, L_{2}\right]$. It follows from Lemma 1.2 that the Hamming distances $\mathrm{HD}, \mathrm{HD}_{1}$, and $\mathrm{HD}_{2}$ of $L, L_{1}$, and $L_{2}$ satisfy $\mathrm{HD}=$ $d^{n}, \mathrm{HD}_{1} \leq d^{n-1}$, and $\mathrm{HD}_{2} \geq(d-1) \cdot d^{n-1}$. Consequently, we can obtain an $\operatorname{IrOA}\left(d^{n+1}, N_{n}, d, 2\right)$ for $d^{n-1}+3 \leq N_{n} \leq \frac{d^{n+1}-1}{d-1}$ by deleting $0 \leq j \leq \frac{1}{d-1}\left[\left(d^{2}-d+1\right) d^{n-1}-3 d+2\right]$ columns from $L$ in the same way as in Theorem 2.12. When $n=1$, we can obtain $\operatorname{IrOA}\left(d^{2}, N_{1}, d, 2\right)$ for $4 \leq N_{1} \leq d+1$ from a known $\mathrm{OA}\left(d^{2}, d+1, d\right.$, 2). When $n \geq 2$, we have $d^{n-1}+3 \leq N_{n} \leq \frac{d^{n+1}-1}{d-1}$, $d^{n}+3 \leq N_{n+1} \leq \frac{d^{n+2}-1}{d-1}$. It can easily be proved that $d^{n}+3 \leq \frac{d^{n+1}-1}{d-1}$, so we obtain $\operatorname{IrOA}(r, N, d, 2)$ for $N \geq d+3$. To cover the gap $N=d+2$, consider the difference scheme $D(2 d, 2 d$, d) satisfying $D(2 d, 2 d, d)^{T}=D(2 d, 2 d, d)$, which exists by Lemma 1.8. Let $L^{\prime}=D(2 d, 2 d, d) \oplus$ (d). Then, $L^{\prime}$ is an OA. It follows from Lemma 1.9 that $L^{\prime}$ has two Hamming distances, $2 d-2$ and $2 d$. Hence, we can obtain $\operatorname{IrOA}\left(2 d^{2}, N_{d}^{\prime}, d, 2\right)$ for $5 \leq N_{d}^{\prime} \leq 2 d$ from Lemma 1.14. As $d+1 \geq 5$ and $2 d>d+3$, there exists an $\operatorname{IrOA}(r, N$, $d, 2$ ) for $N \geq 4$, which leads to the desired 2-uniform states.

Example 2.5. Let $d=4$. When $n=1,2,3, \ldots$, it follows from Theorem 2.13 that we have an $\operatorname{IrOA}\left(4^{2}, N_{1}, 4,2\right)$ for $4 \leq N_{1} \leq 5$, an $\operatorname{IrOA}\left(4^{3}, N_{2}\right.$, $4,2)$ for $7 \leq N_{2} \leq 21$, an $\operatorname{IrOA}\left(4^{4}, N_{3}, 4,2\right)$ for $19 \leq N_{3} \leq 85$, and so on. In addition, we can obtain an $\operatorname{IrOA}\left(32, N_{4}^{\prime}, 4,2\right)$ for $5 \leq N_{4}^{\prime} \leq 8$. Therefore, there exists an $\operatorname{IrOA}(r, N, 4,2)$ and 2-uniform states of $N$ ququarts for every $N \geq 4$.

Construction of 2-uniform states of $N$ qudits with a non-primepower $d \geq 4$ levels

By computing the minimal distance of symmetrical OAs constructed from the orthogonal partition methods in, ${ }^{32}$ we derive the following results.

When $d=6$, there exists an $\operatorname{IrOA}(r, N, 6,2)$ and 2-uniform states of $N$ qudits for $N \geq 5$.

When $d>6(d \neq \equiv 2(\bmod 4))$ is not a prime power, we can obtain an $\operatorname{IrOA}(r, N, d, 2)$ and 2-uniform states of $N$ qudits for $N \geq 4$.

When $d>6(d \equiv 2(\bmod 4))$ is not a prime power, since every $\operatorname{IrOA}\left(r, N^{\prime}, d, 3\right)$ is also an $\operatorname{IrOA}(r, N, d, 2)$, we can obtain an $\operatorname{IrOA}(r, N$, $d, 2$ ) and 2-uniform states of $N$ qudits for $N=7,8$ and $N \geq 11$ from $\operatorname{IrOA}\left(r, N^{\prime}, d, 3\right)$ for $N^{\prime}=8$ and $N^{\prime} \geq 12$ in Theorem 2.8. We also have an $\operatorname{IrOA}(r, N, d, 2)$ for $N=4,6,9,10$. Then, there exists an $\operatorname{IrOA}(r, N$, $d, 2)$ and 2-uniform states of $N$ qudits for $N=4$ and $N \geq 6$. In particular, when $d \geq 14(d \equiv 2(\bmod 4))$ is not a prime power, there exists an $\operatorname{IrOA}\left(d^{2}, 5, d, 2\right)$ and 2 -uniform states of $N$ qudits for $N \geq 4$.
Theorem 2.14. There exists an $\operatorname{IrOA}(r, N, 6,2)$ and 2-uniform states consisting of $N$ six-level systems for every $N \geq 5$. Proof. We can obtain an $\mathrm{OA}(72,6,6,2)=D(12,6,6) \oplus(6)$ in which $D(12,6,6)$ is given by. ${ }^{42}$ As the minimal distance of the $\operatorname{OA}(72,6,6,2)$ is 4 , we can generate two arrays, $\operatorname{IrOA}(72,6,6,2)$ and $\operatorname{IrOA}(72,5,6,2) . \mathrm{By}_{1}{ }^{43} L=$ $\left[(\mathbf{6}) \oplus \mathbf{0}_{12}, D(12,6,6) \oplus(\mathbf{6})\right]$ is an OA with minimal distance at least 4. Thus, we have an $\operatorname{IrOA}(72,7,6,2)$. Using Lemmas 1.6 and 1.7 and the case $u=v$ in Lemma $1.15, \mathrm{OA}(72,5,6,2)$ and any three of its columns, say $\mathrm{OA}(72,5,6,2)$ and $\mathrm{OA}(72,3,6,2)$, give an $\mathrm{OA}(432$, $8,6,2)$ with minimal distance 3 . Thus, we have $\operatorname{IrOA}(432,8,6,2)$. Additionally, by Theorem 2.5 and from $D(12,6,6)$, we obtain an $\operatorname{IrOA}(r, N, 6,2)$ for every $N \geq 9$. Therefore, the desired IrOAs and 2uniform states exist.

Theorem 2.15. For any non-prime-power $d>6$ and $d \not \equiv 2(\bmod 4)$, there exists an $\operatorname{IrOA}(r, N, d, 2)$ and 2-uniform states of $N$ qudits for every $N \geq 4$. Proof. By Lemma 1.3, a difference scheme $D(d, 3, d)$ exists for any $d \geq 3$ and $d \not \equiv 2(\bmod 4)$ and a $D_{3}\left(d^{2}, 4, d\right)$ exists for any $d \geq 2$. Take $L=D(d, 3, d) \oplus$ (d). It follows from Lemma 1.16 that $\mathrm{MD}(L)=2$. Using the $D(d, 3, d)$ and Theorem 2.5, we obtain an $\operatorname{IrOA}(r, N, d, 2)$ for any integer $N \geq 9$. By Lemma 1.17(3), we have three arrays $\operatorname{IrOA}\left(d^{2}, 6, d, 2\right), \operatorname{IrOA}\left(d^{2}, 5, d, 2\right)$, and $\operatorname{IrOA}\left(d^{2}, 4, d, 2\right)$. From $\mathrm{OA}\left(d^{3}, 4, d, 3\right)=D_{3}\left(d^{2}, 4, d\right) \oplus(\mathbf{d}), \operatorname{IrOA}\left(d^{2}, 4, d, 2\right)$, and Lemma 1.18 , we obtain an $\mathrm{OA}\left(d^{3}, 8, d, 2\right)$ with minimal distance at least 4. Therefore, we have two arrays $\operatorname{IrOA}\left(d^{3}, 8, d, 2\right)$ and $\operatorname{IrOA}\left(d^{3}\right.$, $7, d, 2)$. Thus, we have an $\operatorname{IrOA}(r, N, d, 2)$ for $N \geq 4$. The desired 2uniform states follow from Connection 1.1.

Theorem 2.16. For any non-prime-power $d>6$ and $d \equiv 2(\bmod 4)$, there exists an $\operatorname{IrOA}(r, N, d, 2)$ and 2-uniform states of $N$ qudits for $N$ $=4$ and $N \geq 6$. Proof. It follows from Theorem 2.8 that an $\operatorname{IrOA}\left(r, N^{\prime}\right.$, $d, 3$ ) exists for $N^{\prime}=8$ and $N^{\prime} \geq 12$. From Lemma 1.12, we obtain an $\operatorname{IrOA}(r, N, d, 2)$ for $N=7,8$ and $N \geq 11$. By Lemmas 1.3 and 1.16 , an $D_{3}\left(d^{2}, 4, d\right)$ exists for any $d \geq 2$. Thus, we have an $\mathrm{OA}\left(d^{3}, 4, d, 3\right)=$ $D_{3}\left(d^{2}, 4, d\right) \oplus$ (d) with minimal distance 2 . From the $\mathrm{OA}\left(d^{3}, 4, d, 3\right)$ and the case $u=v$ in Lemma 1.15 , we can construct an $\mathrm{OA}\left(d^{4}, 8, d\right.$, 3) with minimal distance at least 4 . Furthermore, from the $\mathrm{OA}\left(d^{4}\right.$, $8, d, 3)$ and the $\mathrm{OA}\left(d^{3}, 4, d, 3\right)$ and Lemma 1.18 we have an $\mathrm{OA}\left(d^{4}\right.$, $12, d, 2)$ with minimal distance at least 6 . Hence we can obtain an $\operatorname{IrOA}\left(d^{4}, N, d, 2\right)$ for $9 \leq N \leq 12$. From the two arrays $\mathrm{OA}\left(d^{3}, 4, d, 3\right)$ and $\operatorname{OA}\left(d^{2}, 2, d, 2\right)$ and Lemma 1.18 , we have an $\operatorname{OA}\left(d^{3}, 6, d, 2\right)$ with minimal distance at least 3 . Hence, it is an $\operatorname{IrOA}\left(d^{3}, 6, d, 2\right)$. It follows from Lemma $1.17(1)$ that the $\operatorname{IrOA}\left(d^{2}, 4, d, 2\right)$ exists. Thus, we have the desired results.

Theorem 2.17. For any non-prime-power $d \geq 14$ and $d \equiv 2(\bmod 4)$, there exists an $\operatorname{IrOA}(r, N, d, 2)$ and 2-uniform states of $N$ qudits for every $N \geq 4$. Proof. This is an immediate consequence of Lemma 1.17(2) and Theorem 2.16.

Entanglement is an outstanding property of multiparty quantum systems that has been identified as an essential component in many fields of the theory of quantum information. In recent years, there has been considerable interest in generating entangled states of high dimensions. Such states can, in principle, contain a great deal of entanglement, which makes them more complicated but offer promising perspectives for quantum applications. Therefore, they are important to quantum communication.

We constructed 3-uniform states before 2-uniform states because we needed to use some results from the former case. That the newly constructed 3-uniform states are 2-uniform follows immediately from the strength of their corresponding IrOAs. We hope that our method for 2- and 3-uniform states will also contribute to demonstrating the existence of $k$-uniform states for $k \geq 4$ in the homogeneous case. 
Table 2. Existence of 2-uniform states of $N$ subsystems with $d \geq 2$ level

\begin{tabular}{lllll}
\hline$N$ & $2 \leq N \leq 3$ & 4 & 5 & $N \geq 6$ \\
$d$ & & & & \\
\hline$d=2$ & - & $?$ & $\mathrm{~b}$ & $\mathrm{a}$ \\
$d=3$ & - & $\mathrm{a}$ & $\mathrm{a}$ & $\mathrm{a}$ \\
$d=4$ & - & $\mathrm{a}$ & $\mathrm{a}$ & $\mathrm{a}$ \\
$d=5$ & - & $\mathrm{a}$ & $\mathrm{a}$ & $\mathrm{a}$ \\
$d=6$ & - & $?$ & $\mathrm{a}$ & $\mathrm{a}$ \\
$d=7$ & - & $\mathrm{a}$ & $\mathrm{a}$ & $\mathrm{a}$ \\
$d=8$ & - & $\mathrm{a}$ & $\mathrm{a}$ & $\mathrm{a}$ \\
$d=9$ & - & $\mathrm{a}$ & $\mathrm{a}$ & $\mathrm{a}$ \\
$d=10$ & - & $\mathrm{a}$ & $?$ & $\mathrm{a}$ \\
$d \geq 11$ & - & $\mathrm{a}$ & $\mathrm{a}$ & $\mathrm{a}$ \\
\hline
\end{tabular}

Table 3. Existence of 3-uniform states of $N$ subsystems with $d \geq 2$ levels

\begin{tabular}{lllllllll}
\hline$N$ & $2 \leq N \leq 5$ & 6 & 7 & 8 & 9 & 10 & 11 & $N \geq 12$ \\
$d$ & & & & & & & & \\
\hline$d=2$ & - & $?$ & $?$ & a & b & a & a & a \\
$d=3$ & - & $?$ & $?$ & a & a & a & a & a \\
$d=4$ & - & a & $?$ & a & a & a & a & a \\
$d=5$ & - & a & $?$ & a & a & a & a & a \\
$d>6$ is a prime power & - & a & a & a & a & a & a & a \\
$d \geq 6$ is not a prime power & - & $?$ & $?$ & a & $?$ & $?$ & $?$ & a \\
\hline
\end{tabular}

\section{DISCUSSION}

The present work not only answers the question proposed by Goyeneche et al. ${ }^{2}$ and provides a positive answer to the interesting problem stated by Huber et al., ${ }^{14}$ but also presents more general results. In Tables 2 and 3, for any given $d \geq 2$, we summarize the precise existence of the states for every value of $N$. In the tables, "a" denotes that a state can be constructed from an IrOA, whereas " $b$ " represents that a state can be constructed with some judicially inserted minus signs. The symbol "-" means that such a state cannot exist by Lemma 1.11, whereas "?" indicates that we have not yet solved the problem.

Tables 2 and 3 indicate that for any given $d \geq 2$ (prime power or non-prime-power), we exhaustively solve the problem regarding an explicit construction of the 2 and 3-uniform states of every $N$ qudits from IrOAs, except for at most five values of $N$. They are closely related to QECCs, multi-unitary permutation matrices, and mutually orthogonal quantum Latin squares and cubes. ${ }^{1}$ Furthermore, for the k-uniform states with symbol "?" in Tables 2 and 3, we consider the following five cases:

(1) The nonexistence of 3-uniform states of seven qubits and 2uniform states of four qubits has already been proved. ${ }^{2,13}$ In Theorem 3.1, we prove the nonexistence of $\operatorname{IrOA}(r, 7,2,3)$ and $\operatorname{IrOA}(r, 4,2,2)$.

(2) The existence of 3-uniform states of six qubits and 3uniform states of $N$ qutrits for $N=6,7$ has been presented in, ${ }^{44}$ whereas the nonexistence of $\operatorname{IrOA}(r, 6,2,3), \operatorname{IrOA}(r, 6,3$, $3)$, and $\operatorname{IrOA}(r, 7,3,3)$ has been proved in Theorem 3.1.

(3) Interestingly, the existence of 2-uniform states of four qudits $(d=6)$ is unknown. It cannot be derived from IrOAs, because there is no $\operatorname{IrOA}(r, 4,6,2)$ by Theorem 3.1. However, 2uniform states of five qudits $(d=6)$ can be constructed from $\operatorname{IrOA}(72,5,6,2)$ by Theorem 2.14.
(4) The 3-uniform state of seven ququints exists, ${ }^{44}$ although the existence of $\operatorname{IrOA}(r, 7,5,3)$ is unknown.

(5) The existence of $\operatorname{IrOA}(r, 7,4,3), \operatorname{IrOA}(r, 5,10,2)$, and $\operatorname{IrOA}(r, N$, $d$, 3) for $N=6,7,9,10,11$ and non-prime-power $d \geq 6$ and their corresponding states remains unresolved.

Theorem 3.1. $\operatorname{IrOA}(r, 7,2,3), \operatorname{IrOA}(r, 7,3,3), \operatorname{IrOA}(r, 6,3,3), \operatorname{IrOA}(r, 4$, $6,2), \operatorname{IrOA}(r, 6,2,3), \operatorname{IrOA}(r, 4,2,2)$, and $\operatorname{IrOA}(r, 5,2,2)$ do not exist. Proof. Assume that there exists an $\operatorname{IrOA}(r, 7,2,3)$. Then, $r \leq 16$ by Lemma 1.12. However, this contradicts the fact that neither $\mathrm{OA}(16$, $7,2,4)$ nor $O A(r, 7,2,3)$ exists for $r<16$ (ref. ${ }^{45}$ ). By the same argument, $\operatorname{IrOA}(r, 7,3,3), \operatorname{IrOA}(r, 6,3,3)$, and $\operatorname{IrOA}(r, 4,6,2)$ do not exist. Assume there exists an $\operatorname{IrOA}(r, 7,3,3)$. Then, $r \leq 81$, but this contradicts the fact that neither $\mathrm{OA}(81,7,3,4)$ nor $\mathrm{OA}(r, 7,3,3)$ exists for $r<81$. Assume that $\operatorname{IrOA}(r, 6,3,3)$ and $\operatorname{IrOA}\left(r^{\prime}, 4,6,2\right)$ exist. Then, $r=27$ and $r^{\prime}=36$, but this contradicts the fact that neither $\mathrm{OA}(27,6,3,3)$ nor $\mathrm{OA}(36,4,6,2)$ exists. Similarly, $\operatorname{IrOA}(r, 6$, $2,3), \operatorname{IrOA}(r, 4,2,2)$ and $\operatorname{IrOA}(r, 5,2,2)$ do not exist.

It is tempting to believe that some of the states obtained in this paper will be good for experimental purposes and help to quantify $^{46}$ the level of entanglement in some multipartite system. The states are also ideal candidates for quantum information protocols and quantum secret sharing. It is expected that remarkable progress will be made in the field of QECCs by application of the results presented herein. As stated in, ${ }^{2}$ there are many open issues to solve regarding the construction and characterization of entanglement in multipartite quantum systems. The results presented in this paper will establish a foundation for solving other open problems, such as the construction of $k$-uniform states of $N$ qudits $(d \geq 2)$ for $k \geq 4$, including the problem stated by Huber et al., ${ }^{14}$ and heterogeneous multipartite systems, ${ }^{17}$ since the proposed construction methods can be suitable for IrOAs of any strength $k \geq 4$ and irredundant mixed orthogonal arrays (IrMOAs). ${ }^{17,32,33}$

In the construction process, we often encounter the problem that some uniform states could have fewer terms or qudits. If the Hadamard conjecture is considered, Theorems 2.9 and 2.10 state that the number of terms in many 2-uniform states could be reduced. Moreover, the following three theorems indicate that some 2-uniform states can have fewer terms. Furthermore, from Lemmas 1.13 and 1.14, there exist some 3-uniform states with fewer terms than those obtained by Theorem 2.1 (for instance, the two 3-uniform states obtained from $\operatorname{IrOA}(40,16,2,3)$ and $\operatorname{IrOA}(48$, $16,2,3)$ by Lemmas $1.13,1.14$, and Theorem 2.1 , respectively). Additionally, the states obtained by Theorem 3.5 have fewer qubits.

Theorem 3.2. There exist 2-uniform states of $N$ qudits with 10 levels for $N=6,7,9,10$ that have fewer terms than the states given by Theorem 2.16. Proof. Let $\mathrm{OA}(200,6,10,2)=[D(20,5,10) \oplus(10)$, (10) $\oplus \mathbf{0}_{20}$, where $D(20,5,10)$ can be found in. ${ }^{42}$ Then, the minimal distance of the $\mathrm{OA}$ is 3 . Therefore, it is an $\operatorname{IrOA}(200$, $6,10,2)$. By exchanging the 16 th and 17 th rows of the $D(40,6,10)$ in, ${ }^{42}$ we obtain the difference scheme $D^{\prime}(40,6,10)$ and an $O A(400$, $7,10,2)=\left[D^{\prime}(40,6,10) \oplus \mathbf{( 1 0 )},(\mathbf{1 0}) \oplus \mathbf{0}_{40}\right]$ with minimal distance 3. Hence, this is $\operatorname{IrOA}(400,7,10,2)$. Utilizing Lemmas 1.6 and 1.7 and the case $u=v$ in Lemma 1.15, we can construct $O A(2000,10$, $10,2)$ with minimal distance 4 from the $O A(200,5,10,2)=D(20,5$, $10) \oplus$ (10) with minimal distance 2 . Then we obtain an $\operatorname{IrOA}(2000$, $10,10,2)$ and an $\operatorname{IrOA}(2000,9,10,2)$. However, from Theorem 2.16, we can only obtain $\operatorname{IrOA}\left(10^{3}, 6,10,2\right), \operatorname{IrOA}\left(10^{4}, 7,10,2\right), \operatorname{IrOA}\left(10^{4}\right.$, $9,10,2)$, and $\operatorname{IrOA}\left(10^{4}, 10,10,2\right)$. Thus, the theorem holds.

Theorem 3.3. For $N=6,9,10$, there are 2-uniform states of $N$ qudits with 14 levels that have fewer terms than those given by Theorem 2.16. Proof. By exchanging the 26th and 27 th rows in the $D(28,5$, 14) from, ${ }^{42}$ we obtain the difference scheme $D^{\prime}(28,5,14)$ and an 
$\mathrm{OA}(392,6,14,2)=\left[D^{\prime}(28,5,14) \oplus \mathbf{( 1 4 )},(\mathbf{1 4}) \oplus \mathbf{0}_{28}\right]$ with minimal distance 3 . Hence, this is $\operatorname{IrOA}(392,6,14,2)$. Furthermore, from an $\mathrm{OA}(392,5,14,2)=D(28,5,14) \oplus(14)$ with minimal distance 2 , Lemmas 1.6 and 1.7, and the case $u=v$ in Lemma 1.15, we can construct an $\mathrm{OA}(5488,10,14,2)$ with minimal distance 4 . Then, we obtain $\operatorname{IrOA}(5488,10,14,2)$ and $\operatorname{IrOA}(5488,9,14,2)$. These IrOAs have fewer rows than $\operatorname{IrOA}\left(14^{3}, 6,14,2\right), \operatorname{IrOA}\left(14^{4}, 9,14,2\right)$, and $\operatorname{IrOA}\left(14^{4}, 10,14,2\right)$ obtained by Theorem 2.16 . Therefore, the result follows from Connection 1.1.

Theorem 3.4. Some uniform states obtained by Lemma 1.17 have fewer terms than those given by Theorems 2.15 and 2.16 for $N=7,8$. Proof. This follows from the fact that some $\operatorname{IrOA}\left(d^{2}, N, d, 2\right)$ obtained by Lemma 1.17 have fewer rows than that given by Theorems 2.15 and 2.16 for $N=7,8$.

Remark 2. The following theorem and Theorems 2.10 and 2.11 indicate that we can find $\operatorname{more} \operatorname{IrOA}(n, N, 2,2)$ than that suggested by Theorem 2.9 using the construction methods for $\mathrm{OA}(n, n-1,2$, 2). Therefore, we can consider the lowest value of $N$ such that $k$ uniform states exist for fixed $r, d$, and $k$.

Theorem 3.5. If an $\mathrm{OA}(r, r-1,2,2)$ is obtained from an MOA $\left(r, I+u, d_{1}^{1} \cdots d_{l}^{1} 2^{u}, 2\right)$ by using the expansive replacement method and $\mathrm{OA}\left(d_{i}, d_{i}-1,2,2\right)$, where $d_{i} \geq 4$ for $i=1, \ldots, l$, then for any $1 \leq j$ $\leq l$, there exists an $\mathrm{OA}\left(r, r-1-\left[\left(d_{1}-1\right)+\cdots+\left(d_{j}-1\right)\right], 2,2\right)$ with a minimal distance that is at least $\frac{1}{2}\left[r-\left(d_{1}+d_{2}+\cdots+d_{j}\right)\right]$. Proof. It follows from Lemma 1.2 that the Hamming distances of the OA $(r, r$ $-1,2,2), \mathrm{OA}\left(d_{i}, d_{i}-1,2,2\right)(i=1, \ldots, l)$ are $\frac{r}{2}, \frac{d_{i}}{2}$, respectively, since they are saturated. For any $1 \leq j \leq l$, deleting the corresponding $d_{1}$ $-1+d_{2}-1+\cdots+d_{j}-1$ columns obtained by the expansive replacement method in ${ }^{12}$ from $O A(r, r-1,2,2)$, we can obtain the desired OA $\left(r, r-1-\left[\left(d_{1}-1\right)+\cdots+\left(d_{j}-1\right)\right], 2,2\right)$

For instance, if $B_{32}$ is an $\mathrm{OA}(32,31,2,2)$, then from the $\mathrm{OA}(64$, $63,2,2)=\left[B_{32} \oplus \mathbf{0}_{2},\left(\mathbf{O}_{32}, B_{32}\right) \oplus(\mathbf{2})\right]$ and using Theorem 2.10, we can obtain an $\operatorname{IrOA}(64, N, 2,2)$ for $19 \leq N \leq 63$. However, from an $\mathrm{OA}\left(64,21,8^{7} 2^{14}, 2\right)$ and Theorem 3.5 and by deleting the seven 8 level columns, we have $\mathrm{OA}(64,14,2,2)$ with minimal distance at least 4. Thus, an $\operatorname{IrOA}(64, N, 2,2)$ exists for $13 \leq N \leq 63$.

In the future, we will study the optimal problems of the uniform states from IrOAs. At first, we will investigate the optimal problems of IrOAs, as they play an important role in uniform states. For some given $N, d, k$, we consider an $\operatorname{IrOA}(r, N, d, k)$ in which $r$ attains its minimum, while we can also consider the lowest value of $N$ such that an $\mathrm{OA}(r, N, d, k)$ exists for fixed $r, d, k$. For some given $r, N, d$, we consider an $\operatorname{IrOA}(r, N, d, k)$ in which $k$ attains its maximum, and we can also consider the highest value of $d$ such that an $\mathrm{OA}(r, N, d$, $k$ ) exists for fixed $r, N, k$.

\section{METHODS}

By means of the construction methods of OAs, we can investigate the properties of the required IrOAs (see "Introduction" section). Using difference schemes, orthogonal partitions, and the Hamming distance, we creatively construct several infinite series of IrOAs. From these IrOAs, we then obtain the 2 and 3-uniform states of almost every $N$ qudits for any given number of levels $d \geq 2$ by means of the link between an IrOA and a $k$ uniform state, which was established by Goyeneche et al. ${ }^{2}$ In particular, a key construction of uniform states of non-prime-power dimensions is due to the orthogonal partition method for recursively constructing OAs, which is new development in combinatorial designs (see 'Results' section). Starting with some special difference schemes and IrOAs, or exploring the expansive replacement method, we can then obtain some uniform states having fewer terms or qudits (see 'Discussion' section).

\section{DATA AVAILABILITY}

The data that support the findings of this study are available from the corresponding author upon reasonable request.

\section{ACKNOWLEDGEMENTS}

This work was supported by the National Natural Science Foundation of China (Grant No. 11571094).

\section{AUTHOR CONTRIBUTIONS}

S.Q.P. proposed the initial idea and goal. S.Q.P. and X.Z. performed the theoretical calculations and contributed to writing the manuscript. S.Q.P., X.Z. and X.L. discussed and analyzed the results. Q.J.Z. programmed to calculate Hamming distances of OAs.

\section{ADDITIONAL INFORMATION}

Supplementary information accompanies the paper on the npj Quantum Information website (https://doi.org/10.1038/s41534-019-0165-8).

Competing interests: The authors declare no competing interests.

Publisher's note: Springer Nature remains neutral with regard to jurisdictional claims in published maps and institutional affiliations.

\section{REFERENCES}

1. Goyeneche, D., Raissi, Z., Martino, S. D. \& Życzkowski, K. Entanglement and quantum combinatorial designs. Phys. Rev. A 97, 062326 (2018).

2. Goyeneche, D. \& Życzkowski, K. Genuinely multipartite entangled states and orthogonal arrays. Phys. Rev. A 90, 022316 (2014).

3. Arnaud, L. \& Cerf, N. J. Exploring pure quantum states with maximally mixed reductions. Phys. Rev. A 87, 012319 (2013).

4. Scott, A. J. Multipartite entanglement, quantum-error-correcting codes, and entangling power of quantum evolutions. Phys. Rev. A 69, 052330 (2004).

5. Bouwmeester, D. et al. Experimental quantum teleportation. Nature 390, 575-579 (1997).

6. Zhao, Z. et al. Experimental demonstration of five-photon entanglement and open-destination teleportation. Nature 430, 54-58 (2004)

7. Riebe, M. et al. Deterministic quantum teleportation with atoms. Nature 429, 734-737 (2004)

8. Roos, C. F. et al. Control and measurement of three-qubit entangled states. Science 304, 1478-1480 (2004).

9. Lo, H. K., Curty, M. \& Qi, B. Measurement-device-independent quantum key distribution. Phys. Rev. Lett. 108, 130503 (2012).

10. Jozsa, R. \& Linden, N. On the role of entanglement in quantumcomputational speed-up. Proc. R. Soc. A 459, 2011-2032 (2003).

11. Gao, J. et al. Experimental machine learning of quantum states. Phys. Rev. Lett. 120, 240501 (2018)

12. Hedayat, A. S., Sloane, N. J. A. \& Stufken, J. Orthogonal Arrays: Theory and Applications. (Springer-Verlag, New York, 1999).

13. Song, C. et al. 10-qubit entanglement and parallel logic operations with a superconducting circuit. Phys. Rev. Lett. 119, 180511 (2017).

14. Huber, F., G:uhne, O. \& Siewert, J. Absolutely maximally entangled states of seven qubits do not exist. Phys. Rev. Lett. 118, 200502 (2017).

15. Wang, X. et al. 18-qubit entanglement with six photons' three degrees of freedom. Phys. Rev. Lett. 120, 260502 (2018).

16. Zha, X., Yuan, C. \& Zhang, Y. Generalized criterion for a maximally multi-qubit entangled state. Laser Phys. Lett. 10, 045201 (2013).

17. Goyeneche, D., Bielawski, J. \& Życzkowski, K. Multipartite entanglement in heterogeneous systems. Phys. Rev. A 94, 012346 (2016).

18. Goyeneche, D., Alsina, D., Latorre, J., Riera, A. \& Życzkowski, K. Absolutely maximally entangled states, combinatorial designs, and multiunitary matrices. Phys. Rev. A 92, 032316 (2015).

19. Yu, Y., Zha, X. \& Wei, Z. Construction of 3-uniform states via orthogonal arrays (in Chinese). Sci Sin-Phys Mech Astron 46, 060301 (2016).

20. Zha, X., Ahmed, I. \& Zhang, Y. 3-Uniform states and orthogonal arrays. Results Phys 6, 26-28 (2016)

21. Werner, R. F. All teleportation and dense coding schemes. J. Phys. A 34 7081-7094 (2001).

22. Rotteler, M. \& Wocjan, P. Equivalence of decoupling schemes and orthogonal arrays. IEEE Trans. Inform. Theory 52, 4171-4181 (2006). 
23. Pang, S., Wang, X., Wang, J., Du, J. \& Feng, M. Construction and count of 1-resilient rotation symmetric Boolean functions. Inf. Sci. 450, 336-342 (2018).

24. Hedayat, A. S., Seiden, E. \& Stufken, J. On the maximal number of factors and the enumeration of 3-symbol orthogonal arrays of strength 3 and index 2. J. Stat. Plann. Inference 58, 43-63 (1997).

25. Zhang, Y., Lu, Y. \& Pang, S. Orthogonal arrays obtained by orthogonal decomposition of projection matrices. Stat. Sin. 9, 595-604 (1999).

26. Zhang, Y., Pang, S. \& Wang, Y. Orthogonal arrays obtained by the generalized Hadamard product. Discret. Math. 238, 151-170 (2001).

27. Pang, S., Yan, R. \& Li, S. Schematic saturated orthogonal arrays obtained by using the contractive replacement method. Comm. Stat. Theory Methods 46, 8913-8924 (2017).

28. Ji, L. \& Yin, J. Constructions of new orthogonal arrays and covering arrays of strength three. J. Comb. Theory Ser. A 117, 236-247 (2010).

29. Yin, J., Wang, J., Ji, L. \& Li, Y. On the existence of orthogonal arrays OA(3;5; $4 n+$ 2). J. Comb. Theory Ser. A 118, 270-276 (2011).

30. Pang, S., Zhu, Y. \& Wang, Y. A class of mixed orthogonal arrays obtained from projection matrix inequalities. J. Inequal. Appl. 2015, 1-9 (2015).

31. Pang, S. \& Chen, L. Generalized Latin matrix and construction of orthogonal arrays. Acta Math. Appl. Sin. 33, 1083-1092 (2017).

32. Pang, S., Lin, X. \& Wang, J. Construction of asymmetric orthogonal arrays of strength $t$ from orthogonal partition of small orthogonal arrays. IEICE Trans. Fundam. E101-A, 1267-1272 (2018).

33. Pang, S., Xu, W., Chen, G. \& Wang, Y. Construction of symmetric and asymmetric orthogonal arrays of strength $t$ from orthogonal partition. Indian J. Pure Appl. Math. 49, 663-669 (2018).

34. Mukerjee, R. \& Wu, C. J. F. On the existence of saturated and nearly saturated asymmetrical orthogonal arrays. Ann. Stat. 23, 2102-2115 (1995).

35. Chen, G. \& Lei, J. Constructions of mixed orthogonal arrays of strength three (in Chinese). Sci Sin Math 47, 545-564 (2017).

36. Hedayat, A. S., Stufken, J. \& Su, G. On difference schemes and orthogonal arrays of strength $t$. J. Stat. Plann. Inference 56, 307-324 (1996).

37. Zhang, Y. On schematic orthogonal arrays of strength two. Ars. Comb. 91, 147-163 (2009).
38. Shen, H. Theory of Combinatorial Designs (in Chinese). (Shanghai Jiao Tong University Press, Shanghai, 2008).

39. Helwig, W., Cui, W., Latorre, J. I., Riera, A. \& Lo, H. K. Absolute maximal entanglement and quantum secret sharing. Phys. Rev. A 86, 052335 (2012).

40. Suen, C., Das, A. \& Dey, A. On the construction of asymmetric orthogonal arrays. Stat. Sin. 11, 241-260 (2001)

41. Solís-Prosser, M. A., Fernandes, M. F., Jiménez, O., Delgado, A. \& Neves, L. Experimental minimum-error quantum-state discrimination in high dimensions. Phys. Rev. Lett. 118, 100501 (2017).

42. Kuhfeld, W. F. Orthogonal Arrays. http://support.sas.com/techsup/technote/ts723. html. Accessed 29 Sept 2018.

43. Wang, J. C. \& Wu, C. F. J. An approach for the construction of asymmetrical orthogonal arrays. J. Am. Stat. Assoc. 86, 450-456 (1991).

44. Huber, F. \& Wyderka, N. Table of Absolutely Maximally Entangled States. http:// www.tp.nt.uni-siegen.de/+fhuber/ame.html. Accessed 16 Mar 2019.

45. Schoen, E. D., Eendebak, P. T. \& Nguyen, M. V. M. Complete enumeration of purelevel and mixed-level orthogonal arrays. J. Comb. Des. 18, 123-140 (2010).

46. Horodecki, M. Entanglement measures. Quantum Inf. Comput. 1, 3-26 (2001).

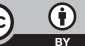

Open Access This article is licensed under a Creative Commons Attribution 4.0 International License, which permits use, sharing, adaptation, distribution and reproduction in any medium or format, as long as you give appropriate credit to the original author(s) and the source, provide a link to the Creative Commons license, and indicate if changes were made. The images or other third party material in this article are included in the article's Creative Commons license, unless indicated otherwise in a credit line to the material. If material is not included in the article's Creative Commons license and your intended use is not permitted by statutory regulation or exceeds the permitted use, you will need to obtain permission directly from the copyright holder. To view a copy of this license, visit http://creativecommons. org/licenses/by/4.0/.

(c) The Author(s) 2019 